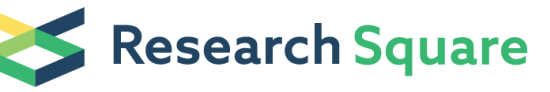

\section{TJCYR ameliorates embryo implantation dysfunction through PI3K/Akt/eNOS signalling to improve endometrial receptivity}

Hong-li Huang

Shanghai University of Traditional Chinese Medicine Yueyang Hospital of Integrated Traditional Chinese Medicine and Western Medicine

\section{Lei Xia}

Shanghai University of Traditional Chinese Medicine Yueyang Hospital of Integrated Traditional Chinese Medicine and Western Medicine

\section{Yan-qiu Xia}

Shanghai University of Traditional Chinese Medicine Yueyang Hospital of Integrated Traditional Chinese Medicine and Western Medicine

\section{Yun-ping Yan}

Shanghai University of Traditional Chinese Medicine Yueyang Hospital of Integrated Traditional Chinese Medicine and Western Medicine

\section{Zhuo-jun Jiang}

Shanghai University of Traditional Chinese Medicine Yueyang Hospital of Integrated Traditional Chinese Medicine and Western Medicine

pei zhao ( $\nabla$ zp1737@sina.com )

Shanghai University of Traditional Chinese Medicine https://orcid.org/0000-0002-8896-6701

\section{Li Dong}

Shanghai University of Traditional Chinese Medicine Yueyang Hospital of Integrated Traditional Chinese Medicine and Western Medicine

\section{Research}

Keywords: Tiao Jing Cu Yun Recipe, endometrial receptivity, embryo implantation dysfunction, biomarker, angiogenesis, PI3K/Akt/eNOS

Posted Date: December 23rd, 2020

DOl: https://doi.org/10.21203/rs.3.rs-132200/v1

License: (c) (i) This work is licensed under a Creative Commons Attribution 4.0 International License. Read Full License 

TJCYR ameliorates embryo implantation dysfunction through

$\mathrm{PI} 3 \mathrm{~K} / \mathrm{Akt} / \mathrm{eNOS}$ signalling to improve endometrial receptivity Hong-li Huang ${ }^{1 \#}$, Lei Xia ${ }^{1 \#}$,Yan-qiu Xia ${ }^{1}$, Yun-ping Yan $^{1}$, Zhuo-jun Jiang ${ }^{1}$, Pei Zhao ${ }^{2 *} \&$ Li Dong ${ }^{1 *}$

${ }^{1}$ Yueyang Hospital of Integrative Chinese and Western Medicine, Shanghai University of Traditional Chinese Medicine, China

${ }^{2}$ The Public Experiment Platform, Shanghai University of Traditional Chinese Medicine, China

*Corresponding Author: Pei Zhao \& Li Dong

Pei Zhao, PhD, The Public Experiment Platform, Shanghai University of Traditional Chinese Medicine, Room 1219, 1200 Cailun Road, Shanghai 201203, China

E-mail: zp1737@sina.com; Phone: +86-13564899026

Li Dong, PhD, Yueyang Hospital of Integrative Chinese and Western Medicine, Shanghai University of Traditional Chinese Medicine, 110 Ganhe Road, Shanghai 200437, China

E-mail: yydongli@163.com; Phone: +86-18930565213

Key words Tiao Jing $\mathrm{Cu}$ Yun Recipe; endometrial receptivity; embryo implantation dysfunction; biomarker; angiogenesis; PI3K/Akt/eNOS 


\begin{abstract}
:
Backgroud: In our clinical practice, we found that Tiao Jing $\mathrm{Cu}$ Yun Recipe (TJCYR), which was composed of Dangshen, Danshen, Danggui, Huangqi, Shudihuang, Bajitian and Yinyanghuo, had obviously enhanced the rate of pregnancy in the women with infertility. Although the application effects are desirable and satisfactory, the therapeutic mechanism of TJCYR remains poorly understood. In this study, we evaluated the effect of TJCYR on embryo implantation dysfunction (EID)-induced damage of endometrial receptivity in mice and investigated the mechanisms underlying the effect.
\end{abstract}

Methods: Pregnant mice were randomly divided into six groups: Control, EID only, Progesterone (Prog)+EID, TJCYR-low-dose+EID, TJCYR-medium-dose+EID, TJCYR-high-dose+EID. Mifepristone was injected to make EID model. On the eighth day of pregnancy, the mice were sacrificed and the number of uterus-implanted blastocysts was counted. On the fourth day of pregnancy, the serum was to analyze the level of hormone by radioimmunoassay, the uterus was to analyze morphology by H\&E and SEM, the combination of immunofluorescence and western blot were to identify the related proteins.

Results: Compared with the EID group, the mice treated with high-dose TJCYR had a greater number of implanted sites, so we choose that dose of TJCYR as treatment in the following study. The mice treatment with 
TJCYR could significantly enhance the level of $\mathrm{P}_{4}$, and inhibite the decrease in the expression of PR that induced by EID. Compared with the EID only, the SEM showed that a marked increase in the number of well-developed pinopodes in the TJCYR treatment group. Except morphological marker, several molecules in relation to pinopodes that could be used as biomarkers. TJCYR abrogated the EID-induced weakened in those biomarkers. Additionally, the vascular density and VEGF were decreased in the EID group, it appeared severe tissue hypoxia, while TJCYR reversed that change. Compared with the control, the p-Akt and p-eNOS were decreased in EID group, accompanied with decline of NO. While TJCYR promoted the activation of Akt and eNOS, to improve the poor microvascular environment of endometrium.

Conclusion: TJCYR has therapeutic potential against poor endometrial receptivity via activation of the PI3K/AKT/eNOS signaling pathway. 


\section{Background}

The incidence of infertility has been one of the health problems worldwide in women ${ }^{[1]}$, which increase the economic, physical, and emotional burden on approximately $9 \%$ of couples worldwide ${ }^{[2]}$. Although access to assisted reproductive technology (ART) is abundant in developed countries and has overcome the majority of infertility causes, success rates have stagnated to around $30 \%{ }^{[3]}$. The successful implantation of embryo is a dynamic process that occurs as a result of interplay among the quality of embryo and the status of endometrium. In the recent years, advances in ART allowed for the selection of high quality embryos, but poor reproductive outcomes remained high. It has been confirmed that $2 / 3$ of in vitro fertilization and embryo transfer (IVF-ET) implantation failures were considered to be due to uterus with decreased endometrial receptivity ${ }^{[4]}$. Endometrial receptivity refers to the finite window in each menstrual cycle in which the endometrium is sufficiently prepared for an implanting blastocyst, therfore improving endometrial receptivity is the key point to rise the pregnancy rate in women with infertility ${ }^{[5-8]}$.

After a long period of research, there are some markers to assess endometrial receptivity, including morphological and biochemical changes. Pinopodes are ultrastructural changes on the apical surface of the luminal epithelium of the uterus. There are also some biochemical 
changes, such as Integrins, leukaemia inhibitory factor, osteopontin and so on, which are synchronous with pinopodes.

Tiao Jing Cu Yun Recipe (TJCYR), which consists of Dangshen, Danshen, Danggui, Huangqi, Shudihuang, Bajitian, Yinyanghuo, is a traditional Chinese medicine that has been widely used as an infertility treatment. Lin et al. found that TJCYR could improve kidney deficiency syndrome of anovulatory infertility patients, regulate the follicular development, and elevate the pregnancy rate. Its actions might be associated with regulating their sex hormones, expressions of ovary local factors such as INHB, ACTA, and FS ${ }^{[9]}$. Huang et al. found that TJCYR can significantly improve the ovulation rate and the reproductive function of endocrine axis in sterile rats caused by androgen ${ }^{[10]}$. Yu et al. reported that Epimedium brevicornu Maxim. and Morinda officinalis How. improved endometrial receptivity in ovulation stimulation (OS) and EID mice through significant improvements in the spatial and temporal expression of pinopodes, accompanied with significantly increase the number of embryonic implantation sites ${ }^{[11]}$. Tao et al. reported that Bushen Huoxue Formula, which is composed of TJCYR, can improve the ACT-INH-FS system in patients with polycystic ovary syndrome of kidney deficiency and blood stasis pattern ${ }^{[12]}$. In our clinic, we found that TJCYR had a good effect on pregnancy, but the pharmacological mechanism of its action is still under investigation. The aim of this study was to document 
the beneficial effect of TJCYR on EID-induced damage of endometrial receptivity in the mice and to investigate the mechanism of action. From this point of view, we have strived to find novel candidates from traditional medicinal herbs for improving endometrial receptivity.

\section{Materials and Methods}

\section{Preparation of TJCYR}

TJCYR is composed of Dangshen, Danshen, Danggui, Huangqi, Shudihuang, Bajitian, and Yinyanghuo in a dry weight ratio of 20:20:20:20:15:12:12. All herbs were supplied by the Shanghai Kangqiao Chinese Medicine Tablet Co., Ltd (Shanghai, China) (Table 1). The decoction pieces mixture was added with water of 4 times volume, boiled 2 times, concentrated using a rotary evaporator, and obtained the equivalent crude content of $2.0 \mathrm{~g} / \mathrm{ml}$. The dosage of TJCYR represents the dry weight of the raw herbs used to produce decoction. 10 times of the normal dosage for adult human was defined as the dosage of TJCYR for mice. Thus, mice in TJCYR group were given $10 \mathrm{~g} / \mathrm{kg} / \mathrm{day}$ of TJCYR.

Table1. Tiao Jing $\mathrm{Cu}$ Yun Recipe (TJCYR) components

\begin{tabular}{lcll}
\hline Chinese & Generic name & Scientific name & Product \\
term & & & lot \\
\hline Dangshen & $\begin{array}{c}\text { Codonopsis pilosula } \\
\text { (Franch.) Nannf. }\end{array}$ & Codonopsis radix & 171011 \\
& Salvia miltiorrhiza & Salviae miltiorrhizae & 170701
\end{tabular}


Bge. $\quad$ radix et rhizoma

Danggui

Angelicae sinensis

170606

Angelica sinensis radix

(Oliv.) Diels

Huangqi

Astragalus

Astragali radix

160829

membranaceus

(Fisch.) Bunge.

Shudihuang Rehmannia glutinosa Rehmanniae radix 170420

(Gaert.) Libosch.ex

praeparata

Fisch.et Mey.

Bajitian $\quad$ Morinda officinalis $\quad$ Morindae officinalis 171219

How.

radix

Yinyanghuo Epimedium brevicornu Epimedii folium 170723

Maxim.

Fingerprinting high-performance liquid chromatography (HPLC) analysis

The phytochemical property of TJCYR was examined by HPLC analysis, comparing it to known compounds that included Calycosin-7-glucoside, Acteoside, Salvianolic acid B, Icariin, Tanshinone IIA. The multi-components of the TJCYR were performed on the 1200 series HPLC device (Agilent Technologies, Santa Clara, CA, USA) equipped with an autosampler (G1329B), thermostatted column compartment (G1316A), quaternary pump (G1311A), photodiode array detector (G1315D), and degasser (G1322A). HPLC was performed on an Apollo 
C18 (4.6×250mm;partical size, $5 \mu \mathrm{m}$; GRACE, Columbia, Maryland, USA) with a mobile phase of acetonitrile (A) $-0.1 \%(\mathrm{v} / \mathrm{v})$ phosphoric acid (B) for gradient elution (0-70 min, 1-40\% A; $70-90$ min, 40-80\% A). The analysis was operated at a flow rate of $1 \mathrm{~mL} / \mathrm{min}$, a column temperature of $30{ }^{\circ} \mathrm{C}$ and a detection wavelength of $260 \mathrm{~nm}$. The injection volume was $10 \mu \mathrm{L}$. The standard solutions and sample were filtered with a $0.45 \mu \mathrm{m}$ membrane filter before subjecting them to HPLC analysis.

\section{Experimental animals}

All experimental protocols were approved by the Animal Care and Use Committee of Shanghai University of Traditional Chinese Medicine (Shanghai, China). Adult female and male Kunming mice (weighing 25-28g) were purchased from Beijing Vital River Laboratory Animal Technology Co, Ltd. (Beijing, China). The female mice were virginal and the male mice had been proven to be fertile. The mice were housed in cages separately with controlled temperature and humidity, $12 \mathrm{~h}$ light-dark periods, and free access to water and a standard diet. Prior to the experiments, all mice were given a period of about 2 days for acclimatization. The two estrous cycles were observed by using vaginal smears in female mice before the treatment.

\section{Treatments and Establishment of EID}

The female mice were mixed with male mice in ratio of 2:1 mating from 19:00 to 07:00 and then checked for the presence of a vaginal plug at 
08:00 the next day. Females presenting vaginal plugs (classified as day 1 of pregnancy, Pd1) were randomly divided into six experimental groups ( $n=20$ for each group), including the Control, EID only, Progesterone (Prog) +EID, TJCYR-low-dose+EID, TJCYR-medium-dose+EID and TJCYR-high-dose+EID. In the Control and the EID only group, the mice were given intragastric administration of physiological saline solution once daily for 4 days, the treatment group were given Prog and TJCYR respectively. Mifepriston (0.1mg/mouse) was subcutaneously injected at Pd4 in the morning, to establish the EID model. Mice were sacrificed on $\operatorname{Pd} 4$ and $\mathrm{Pd} 8$, respectively. The uteri were harvested for the evaluation of endometrial receptivity and the potential mechanism (Figure1A).

\section{Determination of implantation sites number}

In one set of our experiment, the gestational mice were sacrificed on Pd8. On this time, the implantation sites in the uterus were captured easily. And the number of implantation sites was counted.

\section{Radioimmunoassay for the test of $E_{2}$ and $P_{4}$}

Serum sex-steroid levels were determined from the serum of blood collected on Pd4. Anesthesia was administered using $0.4 \%$ sodium pentobarbital. Blood samples were collected from the abdominal aortic under anesthesia. The serum was separated by centrifugation at $3000 \times g$ $\left(4^{\circ} \mathrm{C}\right)$ for $10 \mathrm{~min}$. Serum was stored at $-20^{\circ} \mathrm{C}$. The level of $\mathrm{E}_{2}$ and $\mathrm{P}_{4}$ were 
detected using radioimmunoassay, which followed the instructions of the Estradiol Radioimmunoassay Kit (Beijing north institute of biotechnology, Beijing, China) and Iodine $\left[{ }^{125} \mathrm{I}\right]$ Progesterone Radioimmunoassay Kit (Beijing north institute of biotechnology, Beijing, China) respectively. Histologic analysis using hematoxylin and eosin $(H \& E)$ staining Tissue samples were fixed in $4 \%$ paraformaldehyde overnight at $4{ }^{\circ} \mathrm{C}$, rinsed, and transferred to PBS, followed by paraffin embedding, then serially sectioned at a thickness of $5 \mu \mathrm{m}$ for histologic analysis. Sections were stained with hematoxylin for 15 min and washed with dripping water. The sections were then stained with eosin for 3 min and washed with dripping water. The slides were mounted with Permount TM Mounting Medium (Sinopharm Chemical Reagent Co.,Ltd, Shanghai, China) and then covered with coverslips. Finally, the slides were investigated using an optical microscope (Stemi DV4, Carl Zeiss, Oberkochen, Germany).

\section{Scanning electron microscopy (SEM)}

The uteri were excised and then cut open along the longitudinal axis. The endometrial tissue was rinsed in saline solution for $1 \mathrm{~min}$, fixed in $1.25 \%$ (w/v) glutaraldehyde solution and $1 \%$ osmic acid at $4^{\circ} \mathrm{C}$ for $2 \mathrm{~h}$ respectively. After several washes in buffer, the samples were dehydrated through graded concentrations of ethanol and subsequently dried in a critical-point drier with carbon dioxide, then mounted onto the specimen 
holder and coated with gold palladium. Finally, all specimens were observed under the scanning electron microscope (SU8010, Hitachi Instruments, Tokyo, Japan).

\section{Immunofluorescence staining}

For immunofluorescence assay, the samples were frozen in OCT in liquid nitrogen and were serially frozen-sectioned at $8 \mu \mathrm{m}$ thick. Nonspecific protein were blocked with $5 \%$ fetal bovine serum for $1 \mathrm{~h}$ at room temperature (RT). Then sections were incubated with primary antibodies or fluorescence probe overnight at $4{ }^{\circ} \mathrm{C}$. Such as Estrogen Receptor alpha antibody (ER $\alpha, 1: 200$, Abcam, Cambridge, CB, UK), Estrogen Receptor beta1 antibody (ER $\beta 1$, 1:500, Abcam, Cambridge, CB, UK), Progesterone Receptor antibody (PR, 1:200, Abcam, Cambridge, CB, UK), Integrin alpha V antibody (Integrin $\alpha \mathrm{V}, 1: 200$, Abcam, Cambridge, $\mathrm{CB}$, UK), Integrin beta 3 antibody (Integrin $\beta 3$, 1:100, Abcam, Cambridge, CB, UK), Osteopontin antibody (OPN, 1:500, Abcam, Cambridge, CB, UK), LIF antibody (LIF, Abcam, Cambridge, CB, UK), Tomato Lectin (Lycopersion Esculentum Lectin, LEL, Vector Laboratories, Inc., Burlingame, CA, USA), FITC-MAb1 antibody (HP2 Hypoxyprobe $^{\mathrm{TM}}-1$ Plus Kit, Hypoxyprobe, Inc., Burlington, MA, USA). The next day, the sections were incubated with the Alexa Fluor $488 \mathrm{~nm}$ (green, CST, Danvers, MA, USA) or Alexa Fluor 555 nm (red, CST, 
Danvers, MA, USA) for $1 \mathrm{~h}$ at RT in dark, then stained with DAPI (blue,

Sigma, St.Louis, MO, USA). After rinsed three times with PBST, the slides were mounted with Antifade Polyvinylpyrrolidone Mounting Medium (Beyotime, Shanghai, China) and then covered with coverslips. Finally, images were obtained at $488 \mathrm{~nm}$ or $555 \mathrm{~nm}$ excitation by a Carl Zeiss LSM800 confocal microscope (Carl Zeiss Microscope GmbH, Jena, Germany).

\section{Quantification of NO content}

Nitric Oxide (NO) concentration in uteri tissue was measured with the NO assay kit (Beyotime, Shanghai, China) following the user manual. The quantitative determination of nitrite levels represents NO production. The level of NO was shown in $\mu \mathrm{M} / \mu \mathrm{g}$ protein.

\section{Western blotting analysis}

Uteri tissue protein lysates were separated by $10 \%$ SDS-PAGE and then transferred to PVDF $(0.45 \mu \mathrm{m}$, EMD Millipore, Billerica, MA, USA). The membranes were probed overnight at $4{ }^{\circ} \mathrm{C}$ with primary antibodies against ER $\alpha$ (1:1000, Abcam, Cambridge, CB, UK), ERß1 (1:1000, Abcam, Cambridge, CB, UK), PR (1:1000, Abcam, Cambridge, CB, UK), Integrin $\alpha \mathrm{V}(1: 1000, \mathrm{Abcam}$, Cambridge, CB, UK), Integrin $\beta 3$ (1:1000, Abcam, Cambridge, CB, UK), OPN (1:500, Abcam, Cambridge, CB, UK), LIF (1:1000, Abcam, Cambridge, CB, UK), VEGF (1:1000, 
Proteintech, Rosemont, IL, USA), Akt (1:1000, CST, Danvers, MA, USA), p-Akt (1:1000, CST, Danvers, MA, USA), eNOS (1:1000,Abcam, Cambridge, CB, UK), p-eNOS (1:1000, Proteintech, Rosemont, IL, USA), GAPDH (1:3000, Proteintech, Rosemont, IL, USA), or $\beta$-tubulin $(1: 1000$, CST, Danvers, MA, USA) . The next day, the membranes were washed and incubated with the appropriate secondary antibody for $1 \mathrm{~h}$ at RT. After the final wash, signals were detected using the FluorChem E imaging system (ProteinSimple, San Francisco, CA, USA) and a chemiluminescence detection kit (EMD Millipore). Protein band densities were quantified by using an image analysis system (Alpha View SA, ProteinSimple, San Francisco, CA, USA) and expressed as ratios to GAPDH or $\beta$-tubulin.

\section{Statistical analysis}

Data were analyzed using SPSS21.0, and results were presented as means \pm SEM. Data from the experimental groups were compared by one-way analysis of variance (ANOVA) followed by Tukey's post hoc analysis, and differences were considered significant at $P<0.05$.

\section{Results}

\section{The quality evaluation of TJCYR by HPLC.}

HPLC chromatograms of TJCYR crude sample and the standard substance were seen in Figure 1B. Under this separation condition, the index components of herbs can be separated and quantified. It well 
guaranteed the quality and pharmacodynamics of TJCYR.
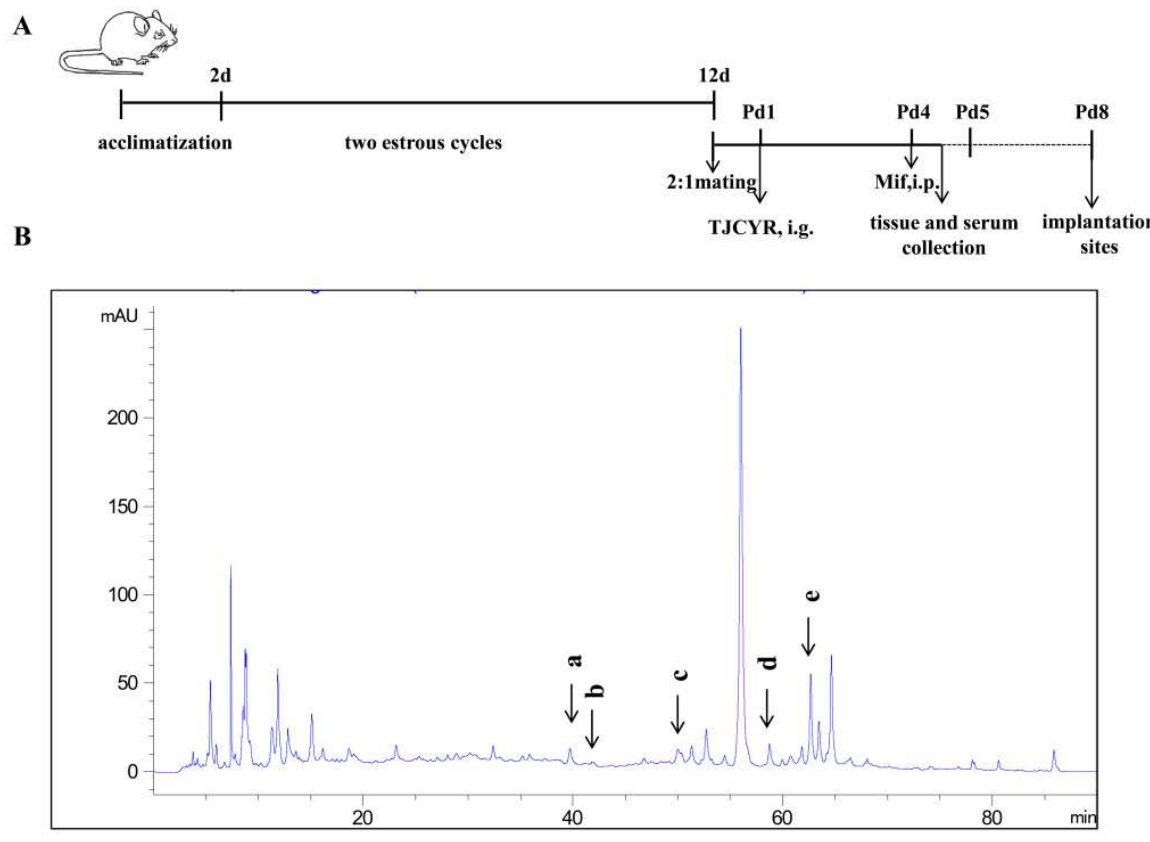

Figure 1. Study scheme and the fingerprinting of TJCYR. (A)

Schematic diagram of experimental protocol; (B) Characteristic fingerprint of TJCYR was analyzed by HPLC. Pd, day of pregnancy; EID, embryo implantation dysfunction; TJCYR, Tiao Jing $\mathrm{Cu}$ Yun Recipe; HPLC, High Performance Liquid Chromatography; a, Calycosin-7-glucoside (standard substance for Astragalus membranaceus (Fisch.) Bunge); b, Acteoside (standard substance for Rehmannia glutinosa (Gaert.) Libosch.ex Fisch.et Mey.); c, Salvianolic acid B (standard substance for Salvia miltiorrhiza Bge.) ; d, Icariin (standard substance for Epimedium brevicornu Maxim.); e, Tanshinone IIA (standard substance for Salvia miltiorrhiza Bge.).

TJCYR was able to protect blastocysts implantation 
Numbers of blastocyst sites were recorded on Pd8. As our results indicated (Figure 2), the mean number of implanted embryos was markedly lower in EID group than in the control group $(P<0.05)$, which suggested successful model. The medium and high-dose of TJCYR treatment increased the implantation numbers compared with the EID only. By comparison, the high-dose of TJCYR dramatically enhances blastocysts implantation, especially, so we choose that dose of TJCYR as treatment in the following study.

A
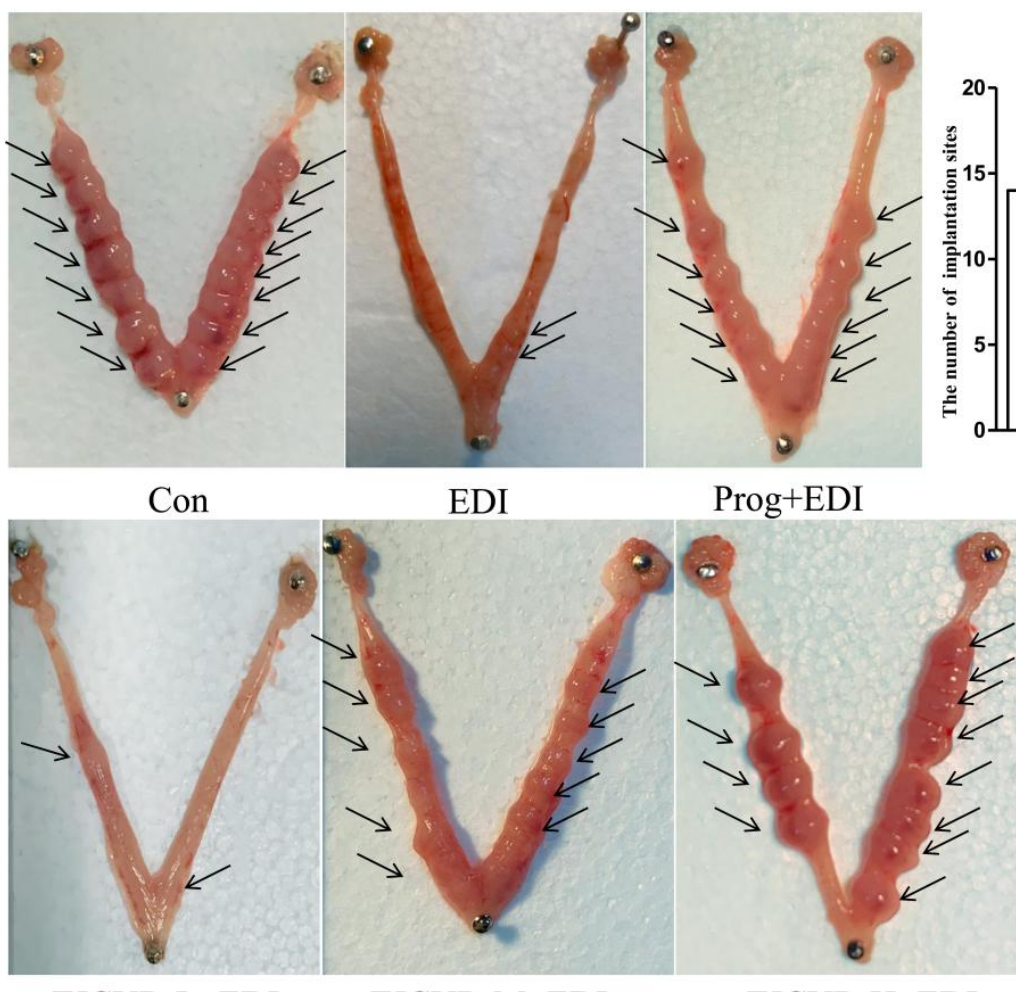

TJCYR-L+EDI TJCYR-M+EDI
B

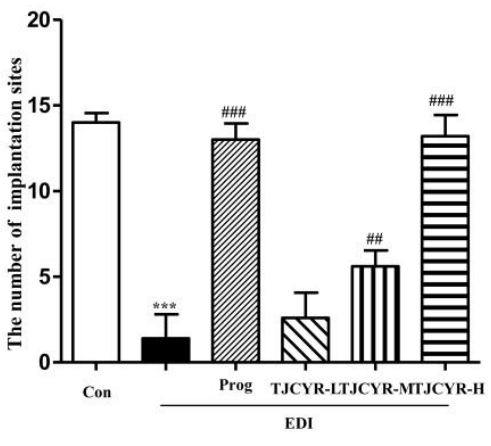

Figure 2. TJCYR incresed the number of implantation sites. (A) A representative photograph showing the number of implantation sites (the arrow pointed) at Pd8; (B) Quantification of implantation sites $(\mathrm{n}=8)$. 
Results are expressed as mean \pm SEM. ${ }^{* * *} P<0.001$ versus Control; ${ }^{\# \#} P<0.05, \quad{ }^{\# \#} P<0.01$ versus EID only. EID, embryo implantation dysfunction; Prog, Progesterone; TJCYR, Tiao Jing $\mathrm{Cu}$ Yun Recipe; TJCYR-H, the high-dose TJCYR; TJCYR-M, the medium-dose TJCYR; TJCYR-L, the low-dose TJCYR.

TJCYR improved EID-induced endometrium morphological changes. As shown in the Figure $3 \mathrm{~A}, \mathrm{H} \& \mathrm{E}$ staining was used to evaluate the pathological changes of endometrium. We discovered that there were little loose endometrial stroma, followed with insufficient glands and vessels in EID only group, which were opposite in mice treated with TJCYR. To further morphologically assess the TJCYR caused changes on EID, we checked the effect of TJCYR on the pinopodes by SEM. As shown in the Figure 3B, in the control group, the sample revealed that the majority of well-developed pinopodes were evenly distributed over the endometrial epithelial surfaces, while EID-induced surfaces only sparsely presented a part of well-developed pinopodes and a few developing pinopodes . However, the restrained expression of pinopodes in the EID group was significantly improved following treatment with TJCYR. The results suggest that TJCYR can improved the morphology and vasculature of endometrium of EID model to a certain extent, which is beneficial to embryo implantation. 
A
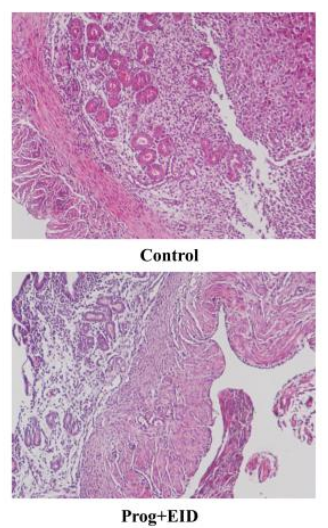

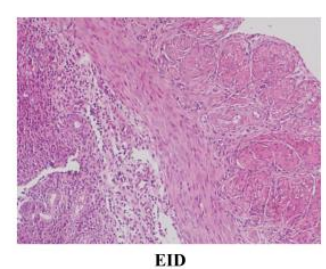

EID

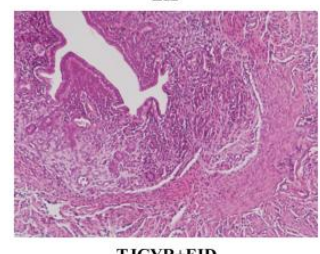

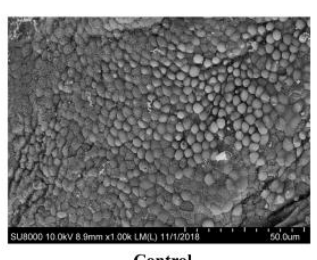

Control

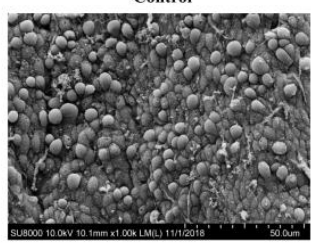

Prog+EID
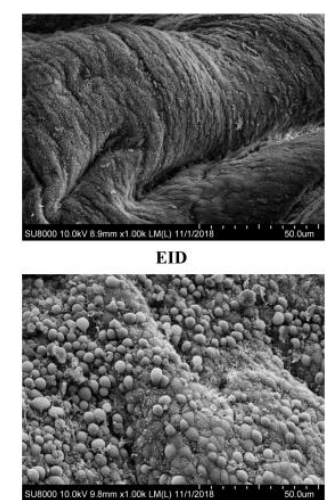

TJCYR+EID

Figure 3. Effect of TJCYR on EID-induced changes in endometrial morphology. (A) H\&E showing pathological changes in endometrium (×200); (B) SEM showing ultrastructure changes in pinopodes.

\section{The effect of TJCYR on hormones and hormone receptors.}

The receptive mouse uterus displays stromal proliferation and epithelial differentiation as the indicator of proper arrangement for blastocyst implantation, which are governed by ovarian hormones, such as: $17 \beta$-estradiol $\left(\mathrm{E}_{2}\right)$ and progesterone $\left(\mathrm{P}_{4}\right)$. And the primary mediators of these $\mathrm{E}_{2}$ and $\mathrm{P}_{4}$-induced events are their receptors- progesterone receptor (PR) and estrogen receptor (ER). In our results, we found that in the EID only group, the level of $\mathrm{P}_{4}$ was obviously lower than those in the Control group, and the expression of PR lowered induced by EID . However, the treatment with TJCYR could reverse those changes (Figure 4) . 


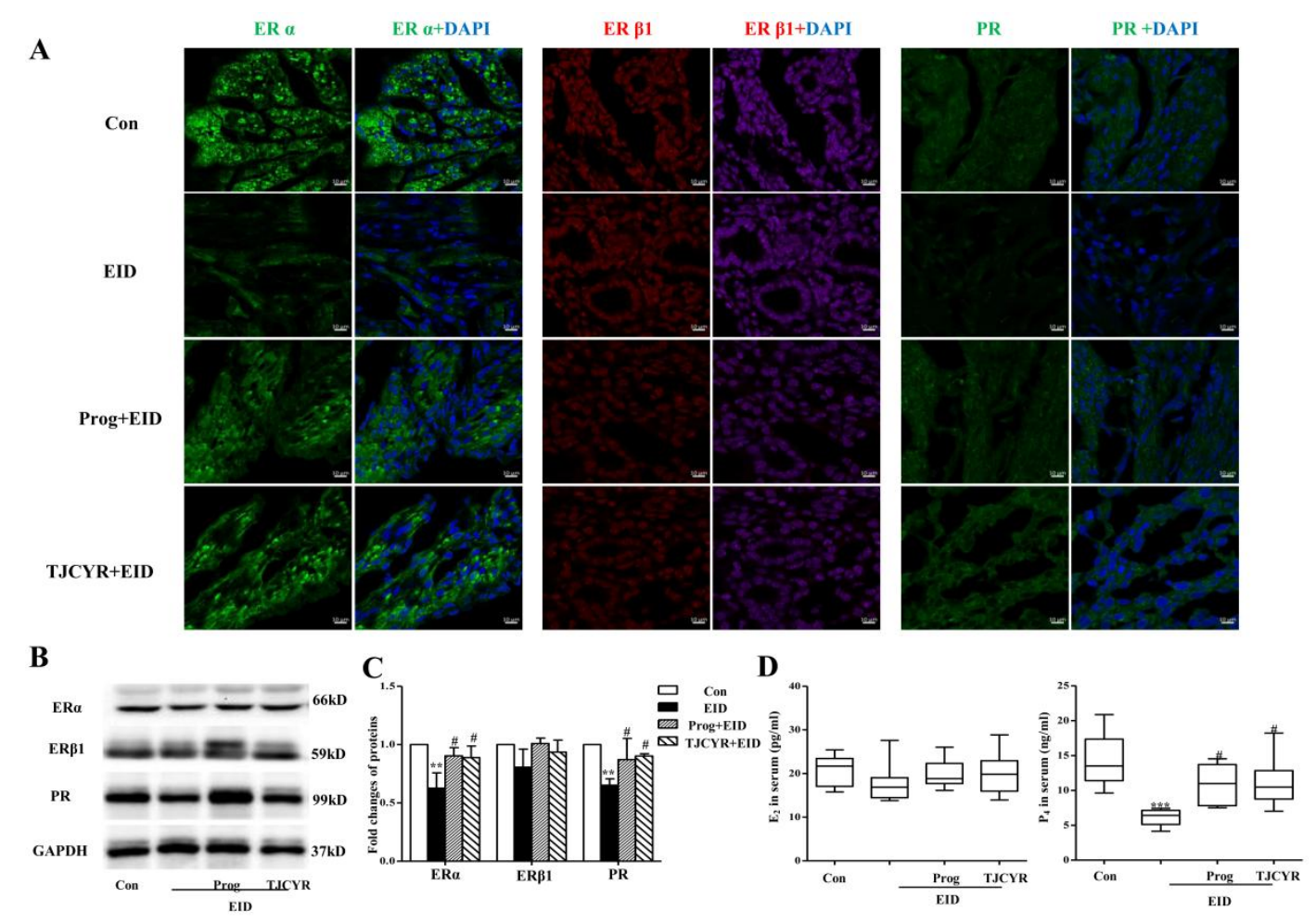

Figure 4. Effect of TJCYR on EID-induced changes in the hormones and receptors. (A) Representative confocal fluorescent images of the uterine sample with ER $\alpha$ (Green), ER $\beta 1$ (Red) and PR (Green), respectively. (B) Protein levels of ER $\alpha, E R \beta 1$ and PR in uterine tissue were determined by Western blotting $(n=3)$; (C) Quantification of protein levels. (D) The level of $\mathrm{E}_{2}$ and $\mathrm{P}_{4}$ in serum were tested by radioimmunoassay. Results are expressed as mean \pm SEM. ${ }^{* * *} P<0.001$, ${ }^{* *} P<0.01$ versus Control; ${ }^{\#} P<0.05$ versus EID only. EID, embryo implantation dysfunction; Prog, Progesterone; TJCYR, Tiao Jing Cu Yun Recipe; $\mathrm{E}_{2}, 17 \beta$-estradiol; $\mathrm{P}_{4}$, progesterone; $\mathrm{ER} \alpha$, Estrogen Receptor alpha; ER $\beta 1$, Estrogen Receptor beta1; PR, Progesterone Receptor.

TJCYR enhanced the related proteins as the markers of endometrial 
receptivity.

There are several molecular markers related to the endometrial receptivity, including Integrin $\alpha \mathrm{V}$, Integrin $\beta 3$, LIF, and OPN. As shown in the Figure 5 , we found that the expression of Integrin $\alpha \mathrm{V}$, Integrin $\beta 3$, LIF, and OPN significantly decreased in EID only group by immunofluorescence and western blot analysis. TJCYR had been found to attenuate the EDI-induced damage through increasing the expression of Integrin $\alpha \mathrm{V}$, Integrin $\beta 3$, LIF, and OPN. These results were consisted with the changes of pinopodes shown in Figure 3B .

A

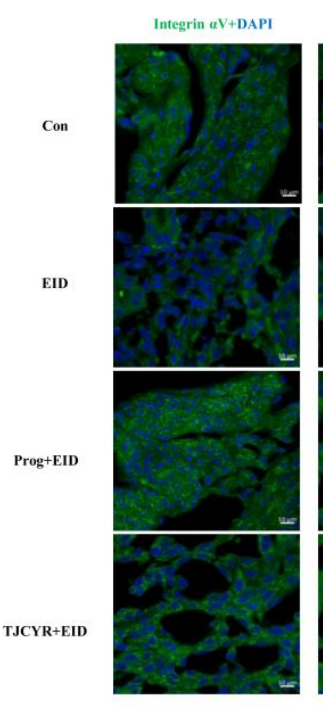

C

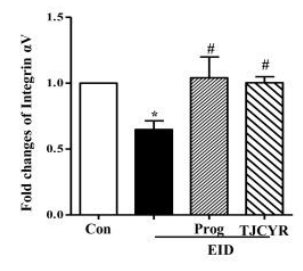

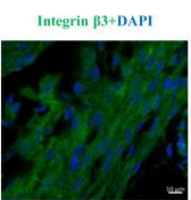
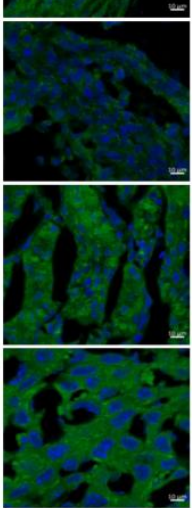
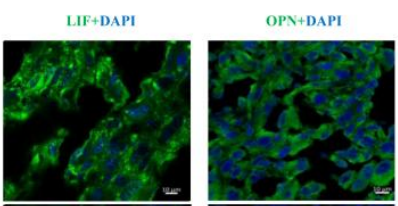

B
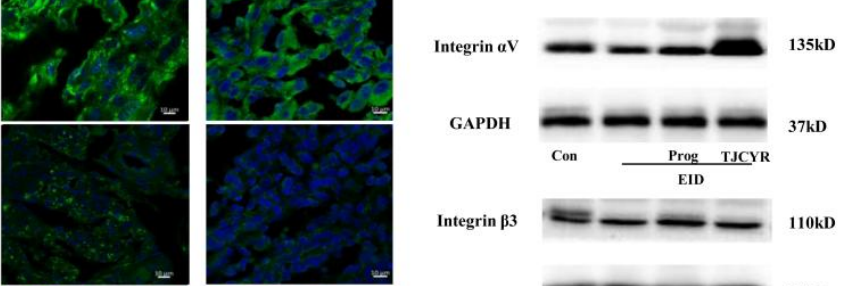

Integrin $\beta 3$

GAPDH
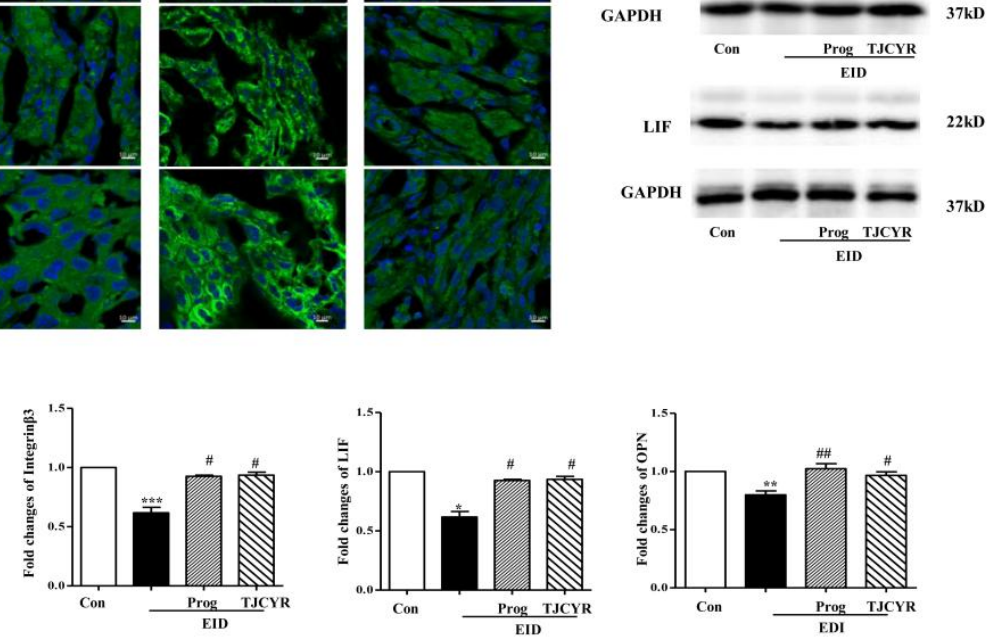

Figure 5. Effect of TJCYR on the biomarkers of endometrial receptivity. (A, D, G and J) Representative confocal fluorescent images of the uterine sample with Integrin $\alpha \mathrm{V}$, Integrin $\beta 3$, LIF and OPN, 
respectively. (B, E, G and $\mathrm{K}$ ) Protein levels of Integrin $\alpha \mathrm{V}$, Integrin $\beta 3$, LIF and OPN in uterine tissue were determined by Western blotting $(\mathrm{n}=3)$; (C, F, I and L) Quantification of protein levels. Results are expressed as mean \pm SEM. ${ }^{*} P<0.05,{ }^{* *} P<0.01,{ }^{* * *} P<0.001$ versus Control; ${ }^{\#} P<0.05$, ${ }^{\# \#} P<0.01$ versus EID only. EID, embryo implantation dysfunction; Prog, Progesterone; TJCYR, Tiao Jing Cu Yun Recipe .

Upregulation of angiogenesis and alleviation of endometrium hypoxia probably contribute to the improvement of endometrial receptivity by TJCYR.

Angiogenesis is one of important biological events which could be involved in implantation. Tomato Lectin is recognized as the most sensitive vessel marker, the fluorescence intensity could reflect the vascular density. Compared with the control group, we discovered that in the EID only group, the vascular density decreased significantly, which was prevented by TJCYR (Figure 6A). Vascular endothelial growth factor (VEGF) is an important mediator of angiogenesis, which has beneficial effects on endometrial receptivity and plays a key role in the embryonic development of mice. As shown in Figure $6 \mathrm{~B}$ and $\mathrm{C}$, the treatment of TJCYR obviously inhibited the decrease of VEGF in mice that subjected to EID, which is consistent with the immunofluorescence analysis (Figure 6A) that showed increased density of vascular.

Hypoxyprobe-1 is the hypoxia marker associated monoclonal and 
polyclonal antibodies that bind to pimonidazole adducts in hypoxia tissue. As shown in the Figure 6D, the fluorescence intensity was increased significantly in mice that subjected to EID only, which suggested serious hypoxia in tissue, while, TJCYR could ameliorat hypoxia

A

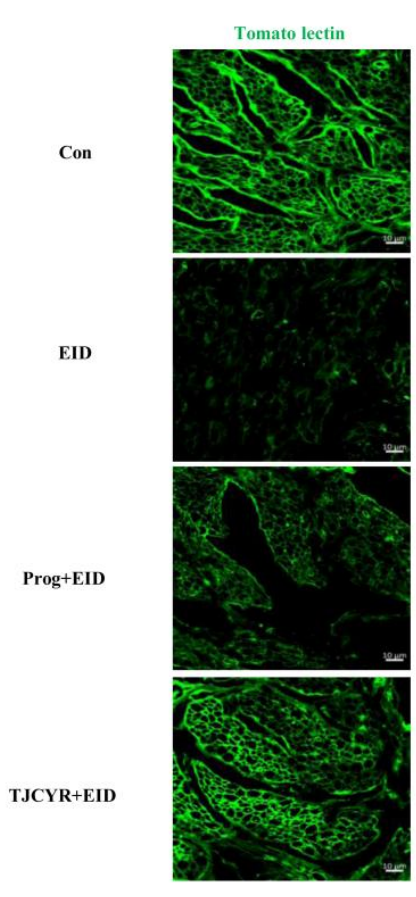

B

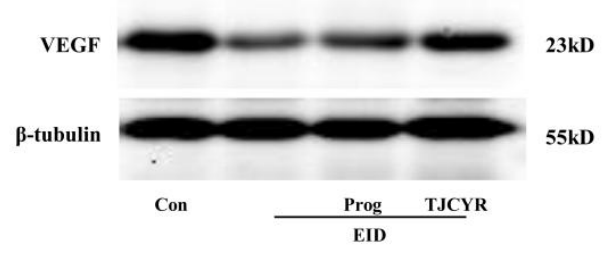

D
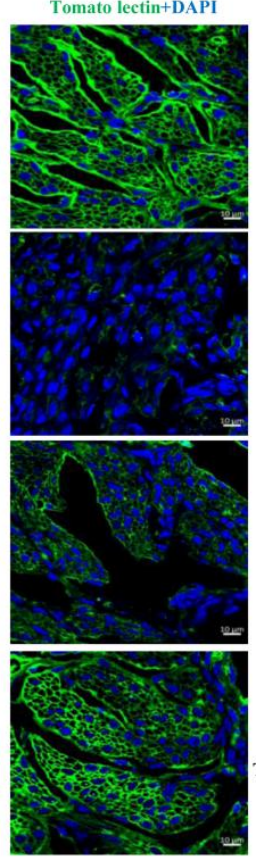

TJCYR+EID

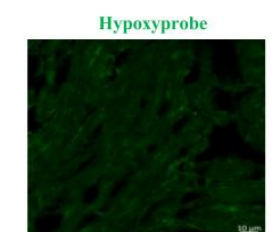

EID
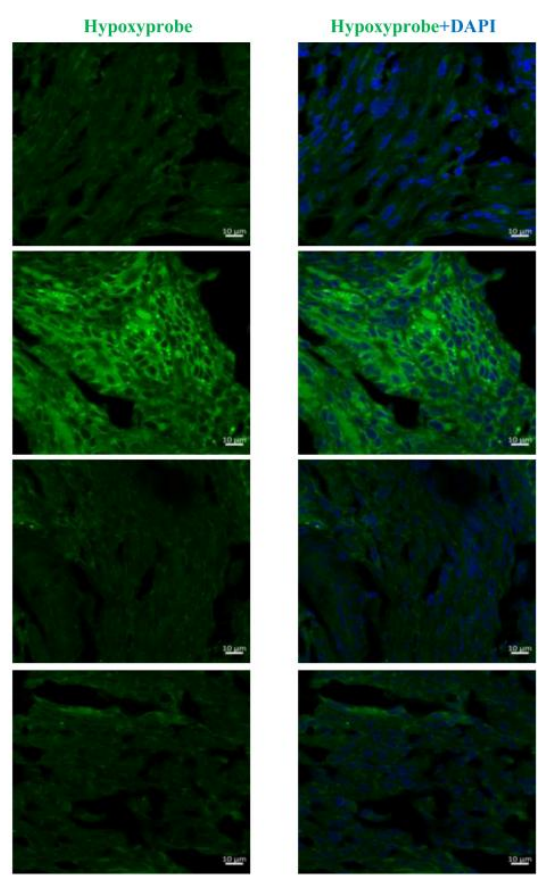

C

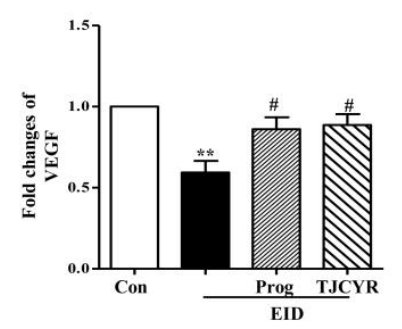

Figure 6. Effect of TJCYR on angiogenesis and hypoxia. (A)

Representative confocal fluorescent images of the uterine sample with Tomato Lectin (Green) and DAPI (Blue); (B) Protein levels of VEGF in uterine tissue were determined by Western blotting $(\mathrm{n}=3) ; \quad(\mathrm{C})$ Quantification of protein levels. Results are expressed as mean \pm SEM; (D) Representative confocal fluorescent images of the uterine sample 
with Hypoxyprobe (Green) and DAPI (Blue). ${ }^{*} P<0.05,{ }^{* *} P<0.01$ versus Control; ${ }^{\#} P<0.05$ versus EID only. EID, embryo implantation dysfunction; Prog, Progesterone; TJCYR, Tiao Jing Cu Yun Recipe.

TJCYR improved angiogenesis through PI3K/Akt/eNOS signaling pathway

The pathway of PI3K/Akt/eNOS is the downstream of VEGF, which participated in angiogenesis. Here, we found that the expressions of p-Akt and p-eNOS were all reduced in the EID only group compared to the control group, accompanied with the decline of NO. While, all of these decreased expressions were restored by the treatment of TJCYR(Figure 7). These data suggested that TJCYR stimulated PI3K/Akt/eNOS signaling pathway, which was deactivated by EID.

A

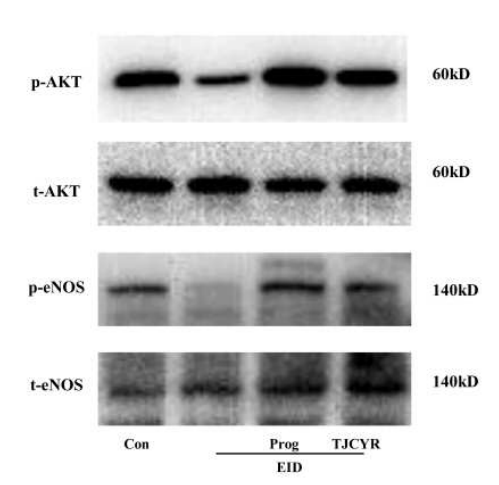

B

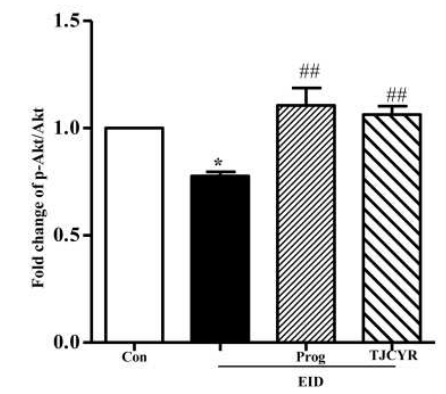

C
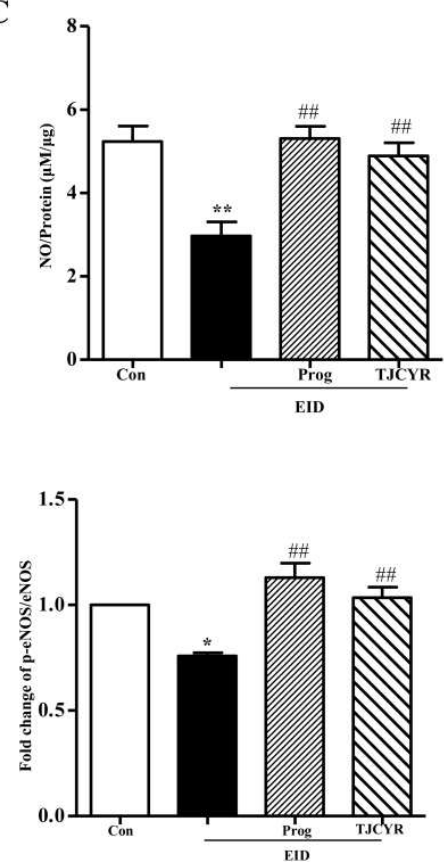

Figure 7. Effect of TJCYR on PI3K/Akt/eNOS signaling pathway. (A)

Protein levels of PI3K/Akt/eNOS signaling pathway in uterine tissue 
were determined by Western blotting $(n=3)$; (B) Quantification of protein levels. Results are expressed as mean $\pm \mathrm{SEM}$; (C) NO production was showed by NO/protein $(n=10)$. Results are expressed as mean \pm SEM. ${ }^{*} P<0.05,{ }^{* *} P<0.01$ versus Control; ${ }^{\#} P<0.01$ versus EID only. EID, embryo implantation dysfunction; Prog, Progesterone; TJCYR, Tiao Jing $\mathrm{Cu}$ Yun Recipe; NO, nitric oxide.

\section{Discussion}

For successful embryo implantation to be achieved, it is a highly orchestrated process that involves blastocyst-uterine interactions. While blastocyst quality has been extensively studied, equally as important is the preparation of the receptive endometrium, or more precisely, it is endometrial receptivity. By definition, endometrial receptivity is "that period of endometrial maturation during which the trophectoderm of the blastocyst can attach to the endometrial epithelial cells and subsequently proceed to invade the endometrial stroma and vasculature" ${ }^{\text {[13] }}$. Therefore, improving endometrial receptivity is the key point to rise the pregnancy rate.

In mice, the implantation window extends from the morning of Pd4 to the end of $\mathrm{Pd} 5^{[14]}$. The use of mifepristone for both planned and emergency contraception has been widely accepted ${ }^{[15]}$. And mifepristone played a role in inhibiting development and maturation of endometrium, hence affecting embryo implantation in mice, as well as for terminatin of 
pregnancy $^{[16]}$. In our study, we used the EID mice model by treatment with mifepristone, and collected samples between 21:00 and 22:00 on $\operatorname{Pd} 4$ (as show in Figure 1A).

The endometrium is a unique tissue which undergoes dramatic and rapid changes throughout the menstrual cycle. It progresses through specific stages in response to ovarian hormones. The receptive mouse uterus displays stromal proliferation and epithelial differentiation as an indicator of proper arrangement for blastocyst implantation ${ }^{[17]}$, which is governed by ovarian hormones. In another word, increased production of ovarian hormones $\mathrm{E}_{2}$ and $\mathrm{P}_{4}$ provides the uterus with a capacity of blastocyst attachment pre-decidualization. Circulating $\mathrm{P}_{4}$ levels rises on $\mathrm{Pd} 3$ due to its production from newly-formed corpus luteum. Elevated level of $\mathrm{P}_{4}$ takes over $\mathrm{E}_{2}$ and becomes a dominant ovarian hormone on $\mathrm{Pd} 4$, increased $\mathrm{P}_{4}$ promoted stromal proliferation and inhibited $\mathrm{E}_{2}$-induced epithelial proliferation. $\mathrm{E}_{2}$ and $\mathrm{P}_{4}$ exerted their respective functions through ER and PR. We found that, compared with EID only, TJCYR could obviously enhance the level of $\mathrm{P}_{4}$ and the corresponding receptorPR, while the influence of $E_{2}$ and $E R$ were little. We thought the difference of influence on $\mathrm{E}_{2}$ and $\mathrm{P}_{4}$ was due to EID model. In our study, mifepristone was prepared for EID model, which has a strong affinity with PR and competitively inhibits the effect of $\mathrm{P}_{4}$. So the regulation of $\mathrm{P}_{4}$ maybe obvious after treatment with TJCYR. 
The pinopodes are membrane protrusions on the apical surface of luminal epithelium. The pinopodes play the key role in the initial stage of implantation by promoting attachment of the embryo, because they prevent the cilia from sweeping off the blastocyst as well as promoting withdrawal of uterine fluid and closure of the uterine cavity ${ }^{[5,18]}$. The pinopodes have been considered as the characteristic morphologic markers to assess endometrial receptivity and to locate the implantation window $^{[16,19]}$. In our study, the specimens were examined by scanning electron microscopy (SEM) for the detection of pinopodes. The result revealed that TJCYR could enhance the number of fully developed pinopodes, accompany with reduced distribution of cilia, which may provide nutrients for embryo and enable its attachment to the uterine endometrium.

The pregnancy is a versatile and dynamic process for the implanting embryo and the endometrial epithelium, which requires a series of rather complicated and synchronous morphological and biochemical changes. Appearance of pinopodes is consistent with the expression of several molecular markers of endometrial receptivity, such as Integrin, leukaemia inhibitory factor (LIF), and osteopontin (OPN). It is meaningful to analyze pinopodes and the molecular biomarkers together, so our further studies exploring the effect of TJCYR on the expression of Integrin, LIF, and OPN. Integrins are cell surface receptors that are involved in 
cell-to-cell and extracellular matrix adhesion. Some Integrins increase during the implantation window. There are many isoforms of integrins in mammals, only three isoforms $\alpha 1 \beta 1, \alpha 4 \beta 1$ and $\alpha \mathrm{V} \beta 3$ are found to be particularly involved in implantation, and $\alpha \mathrm{V} \beta 3$ is seemingly playing more conspicuous roles than others, especially ${ }^{[20]}$. The Integrin $\alpha \mathrm{V} \beta 3$ is a potential receptor for blastocyst attachment and is localized on pinopodes ${ }^{[18]}$. Some studies revealed that the blockade of the Integrin $\alpha \mathrm{V} \beta 3$ or lack of Integrin $\beta 3$ may be related to unexplained infertility ${ }^{[21,22]}$. The role of Integrins in the implantation process had been reached extensively. Several studies had identified an association between Integrin expression and female fertility, which suggested the potential use of Integrins as markers of uterine receptivity ${ }^{[23,24]}$. LIF is a member of the interleukin-6 family of cytokines, which plays a critical role in implantation and pinopodes release secretory vesicles that contain LIF in uterine lumen to enable trophoblast invasion and also affect on immune tolerance during implantation ${ }^{[18,25]}$. Some studies ${ }^{[26,27]}$ showed that the deletion or mutation of LIF reduced implantation failure. Other studies ${ }^{[28,29]}$ reported that endometrial LIF and receptor were higher around the time of implantation in fertile women compared with unexplained infertile women. Other finding ${ }^{[30]}$ also showed that LIF may maintain the proper development of the endometrium and implantation receptivity by regulating downstream target genes. OPN is one of the co-factors involved in cell adhesion and 
invasion during the implantation process, and it is an acidic member of the small integrin- binding ligand family of proteins ${ }^{[31]}$, had been shown to be maximally expressed in the epithelial layer in human, mouse and rabbit uterine. OPN was an important constituent of the uterus during pregnancy ${ }^{[31-33]}$. In this study, the EID group showed histopathological lesions characteristic of pinopodes, as well as low expression of Integrin $\alpha \mathrm{V}$, Integrin $\beta 3$, LIF, and OPN. While the expression of these biochemical markers were significantly increased in mice treated with TJCYR. These data strongly promised a repairment of TJCYR in endometrial receptivity. During the window of implantation, a rich vascular network is necessary to supply nutrients and oxygen. Proper endometrial vascular development and maintenance are crucial for successful pregnancy. Vascular endothelial growth factor (VEGF) has a predominant role in successful implantation and maintenance of pregnancy by increasing vascular permeability or forming vascular network. The expression of VEGF and its receptor were confirmed in early pregnancy. It was reported that VEGF knock-out mice do not produce viable offspring ${ }^{[34]}$. The expression of VEGF increased significantly during implantation windows, which suggested that VEGF promotes angiogenesis and the establishment of capillary network, further improved endometrial receptivity and promoted embryo implantation. Otherwise, deficiency of VEGF may induce the reduction of angiogenesis on implantation site, then lead to miscarry ${ }^{[35]}$. 
In this study, we found that there was a poor vascular network in the mice treated with EID only, accompany with serious hypoxia. Here we found that treatment with TJCYR significantly inhibited decline in density of vessel and expression of VEGF in EID only group, and improved endometrial blood circulation, further prevented the hypoxia. All the data showed that VEGF regulated angiogenesis and built endometrial microenvironment for embryo implantation. As we all know, VEGF is an angiogenesis accelerator, which could activate the PI3K/Akt/eNOS pathway. Here, we found that the expressions of p-Akt and p-eNOS were all reduced in the EID mice compared to the control group, and all of these decreased expressions were restored by the treatment of TJCYR. And the activated eNOS could produce nitric oxide (NO). NO, as an endogenous vasodilator, could increase the vascular dilatation and permeability at the implantation site, further to provide safeguard for embryo implantation and pregnancy maintenance.

\section{Conclusion}

TJCYR can activate the PI3K/AKT/eNOS signaling pathway, promote angiogenesis, and increase the endometrial receptivity, which were beneficial to the implantation of embryos.

\section{Abbreviations}


TJCYR: Tiao Jing Cu Yun Recipe; EID: embryo implantation dysfunction; Prog: Progesterone; Pd: day of pregnancy; HPLC: high-performance liquid chromatography; HE: Hematoxylin-eosin; SEM: scanning electron microscopy; ER $\alpha$ : Estrogen Receptor alpha; ER $\beta 1$ : Estrogen Receptor beta1; PR: Progesterone Receptor; Integrin $\alpha \mathrm{V}$ : Integrin alpha V; Integrin $\beta 3$ : Integrin beta 3; LIF: leukaemia inhibitory factor; OPN: osteopontin; VEGF: Vascular endothelial growth factor; p-Akt: Phospho-Akt; p-eNOS: Phospho- eNOS ; NO: nitric oxide.

\section{Acknowledgements}

Not applicable.

\section{Authors' contributions}

LD conceived and designed the experiments. LX, Y-QX, Y-PY and Z-JJ performed the experiments. $\mathrm{H}-\mathrm{LH}$ and $\mathrm{PZ}$ analyzed the data. $\mathrm{PZ}$ wrote the paper. LD provided revision. Hong-li Huang and Lei Xia are equal contributors.

All authors read and approved the final manuscript.

\section{Funding}

This study was funded by the project of Shanghai Science and Technology Committee (NO: 16401931900), Natural Science Foundation of Shanghai (NO: 16ZR1438000), “Three-year" Development Project of Shanghai Shenkang Hospital Development Center (NO: 16CR3040A), the Budget Research Project of Shanghai Education Commission (NO: 
2019LK091).

\section{Availability of data and materials}

The data used to support the findings of this study are available from the corresponding author upon request.

\section{Ethics approval and consent to participate}

All experimental protocols were approved by the Animal Care and Use Committee of Shanghai University of Traditional Chinese Medicine (Shanghai, China).

\section{Consent for publication}

The manuscript is approved by all authors for publication.

\section{Competing interests}

The authors declare that they have no conflict of interest.

\section{References}

[1]Harper JC, Aittomaki K, Borry P, Cornel MC, et al. Recent developments in genetics and medically assisted reproduction: from research to clinical applications. Eur J Hum Genet 2018; 26:12-23. [2]Szamatowicz M. Assisted reproductive technology in reproductive medicine- possibilities and limitation. Ginekol Pol 2016; 87:820-823.

[3]Norwitz ER, Schust DJ, Fisher SJ. Implantation and the survival of early pregnancy. N Engl J Med 2001; 345:1400-1408.

[4]Surrey ES, Schoolcraft WB. Does surgical management of endometriosis within 6 months of an in vitro fertilization- embryo transfer 
cycle improve outcome. J Assist Reprod Genet 2003; 20:365-370.

[5]Matsumoto H. Molecular and cellular events during blastocyst implantation in the receptive uterus: clues from mouse models. J Reprod Dev 2017; 63:445-454.

[6]Cha J, Sun X, Dey SK. Mechanisms of implantation: strategies for successful pregnancy. Nat Med 2012; 18:1754-1767.

[7]Boivin J, Bunting L, Collins JA, et al. International estimates of infertility prevalence and treatment-seeking: potential need and demand for infertility medical care. Hum Reprod 2007; 22:1506-1512.

[8]Miravet-Valenciano JA, Rincon-Bertolin A, Vilella F, et al. Understanding and improving endometrial receptivity. Curr Opin Obstet Gynecol 2015; 27:187-192.

[9]Lin Y, Dong L. Treatment of anovulatory infertility with shen deficiency syndrome by ZHU's Tiaojing Cuyun Recipe: a clinical evaluation. Chin J Integr Trad West Med 2015; 35:1181-1185.

[10]Huang H, Lin Y, Zhu N, et al. Effect of TiaojingZhuyun Recipe on ovulation induction in androgen-causing sterile rats. J Shanghai Univ Tradit Chin Med 2015; 29:61-63.

[11]Yu N, Yan W, Wang Y, et al. Effect of Zhuyun recipe on endometrial pinopode expression in mice with embryonic implantation dysfunction and ovulation stimulation. Exp Ther Med 2014; 9:488-492.

[12]Tao J, Dong L. Effect of Bushen Huoxue Formula on ACT-INH-FS 
system in patients with polycystic ovary syndrome of the kidney deficiency and blood stasis pattern. Shanghai J Tradit Chin Med 2014; 48:58-61.

[13]Lessey BA, Young SL. What exactly is endometrial receptivity? Fertil Steril 2019;111:611-617.

[14]Yoshinaga K. A sequence of events in the uterus prior to implantation in the mouse. J Assist Reprod Genet 2013; 30:1017-1022.

[15]Basu R, Gundlach T, Tasker M. Mifepristone and misoprostol for medical termination of pregnancy: the effectiveness of a flexible regimen. J Fam Plann Reprod Health Care 2003; 29:139-141.

[16]Huang DM, Nardo LG, Huang GY, et al. Effect of a single dose of mifepristone on expression of pinopodes in endometrial surface of mice. Acta Pharmacol Sin 2005; 26: 212-219.

[17]Hirota Y. Progesterone governs endometrial proliferationdifferentiation switching and blastocyst implantation. Endoc J 2019; 66:199-206.

[18]Rarani FZ, Borhani F, Rashidi B. Endometrial pinopode biomarkers: Molecules and microRNAs. J Cell Physiol 2017; 233:445-454.

[19]Nikas G, Makrigiannakis A. Endometrial pinopodes and uterine receptivity. Ann N Y Acad Sci 2003; 997:120-123.

[20]Raheem KA. Cytokines, growth factors and macromolecules as mediators of implantation in mammalian species. Int J Vet Sci Med 2017; 
19:S6-S14.

[21]Illera MJ, Cullinan E, Gui Y, et al. Blockade of the alpha (V)beta(3) integrin adversely affects implantation in mouse. Biol Reprod 2000; 62:1285-1290.

[22]Germeyer A, Savaris RF, Jauckus J, et al. Endometrial beta3 integrin profile reflects endometrial receptivity defects in women with unexplained recurrent pregnancy loss. Reprod Biol Endocrinol 2014 ; $12: 53-57$

[23]Lessey BA, Damjanovich L, Coutifaris C, et al. Integrin adhesion molecules in the human endometrium: correlation with the normal and abnormal menstrual cycle. J Clin Invest 1992; 90:188-195.

[24]Coughlan C, Sinagra M, Ledger W, et al. Endometrial integrin expression in women with recurrent implantation failure after IVF-ET and its relationship to pregnancy outcome. Fertil Steril 2013; 100:825-830.

[25]Kabir-Salmani M, Nikzad H, Shiokawa S, et al. Secretory role for human uterodomes (pinopods): secretion of LIF. Mol Hum Reprod 2005; 11:553-559.

[26]Achache H, Revel A. Endometrial receptivity markers, the journey to successful embryo implantation. Hum Reprod Update 2006; 12:731-46.

[27]Song H, Lim H, Das SK, et al. Dysregulation of EGF family of growth factors and COX-2 in the uterus during the preattachment and 
attachment reactions of the blastocyst with the luminal epithelium correlates with implantation failure in LIF-deficient mice. Mol Endocrinol 2000; 14:1147-1161.

[28]AghajanovaL. Leukemia inhibitory factor and human embryo implantation. Ann N Y Acad Sci 2004; 1034:176-183.

[29]Dimitriadis E, Stoikos C, Stafford-Bell M, et al. Interleukin-11, IL-11 receptoralpha and leukemia inhibitory factor are dysregulated in endometrium of infertile women with endometriosis during the implantation window. J Reprod Immunol 2006; 69:53-64.

[30]Cheng JG, Chen JR, Hernandez L, et al. Dual control of LIF expression and LIF receptor function regulate Stat3 activation at the onset of uterine receptivity and embryo implantation. Proc Natl Acad Sci USA 2001; 98:8680-8685.

[31]Johnson GA, Burghardt RC, Bazer FW, et al. Osteopontin: roles in implantation and placentation. Biol Reprod 2003; 69:1458-1471.

[32]Kang YJ, Forbes $K$, Carver $J$, et al. The role of the osteopontin-integrin $\alpha v \beta 3$ interaction at implantation: functional analysis using three different in vitro models. Hum Reprod 2014; 29: 739-749.

[33]Altmae S, Martinez-Conejero JA, Salumets A, et al. Endometrial gene expression analysis at the time of embryo implantation in women with unexplained infertility. Mol Hum Reprod 2010; 16:178-187.

[34]Licht P, Russu V, Lehmeyer S, et al. Cycle dependency of intrauterine 
vascular endothelial growth factor levels is correlated with decidulization and corpus luteum function. Fertil Steril 2003; 80:1228-1233.

[35]Lash GE, Innes BA, Drury JA, et al. Localization of angiogenic growth actors and their receptors in the human endometrium throughout the menstrual cycle and in recurrent miscarriage. Hum Reprod 2012; 27:183-195. 


\section{Figures}

A
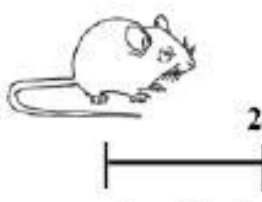

acclimatization

$2 \mathrm{~d}-12 \mathrm{~d}$

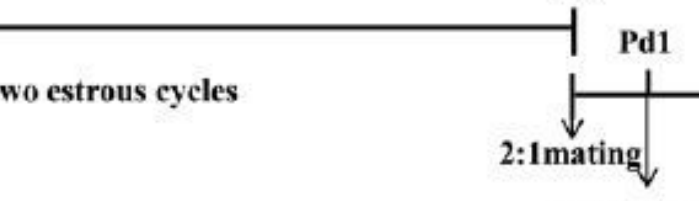

B

TJCYR, i.g.

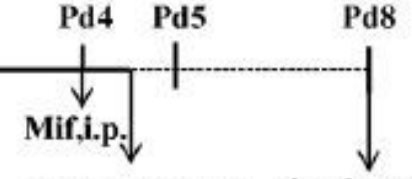

tissue and serum implantation collection sites

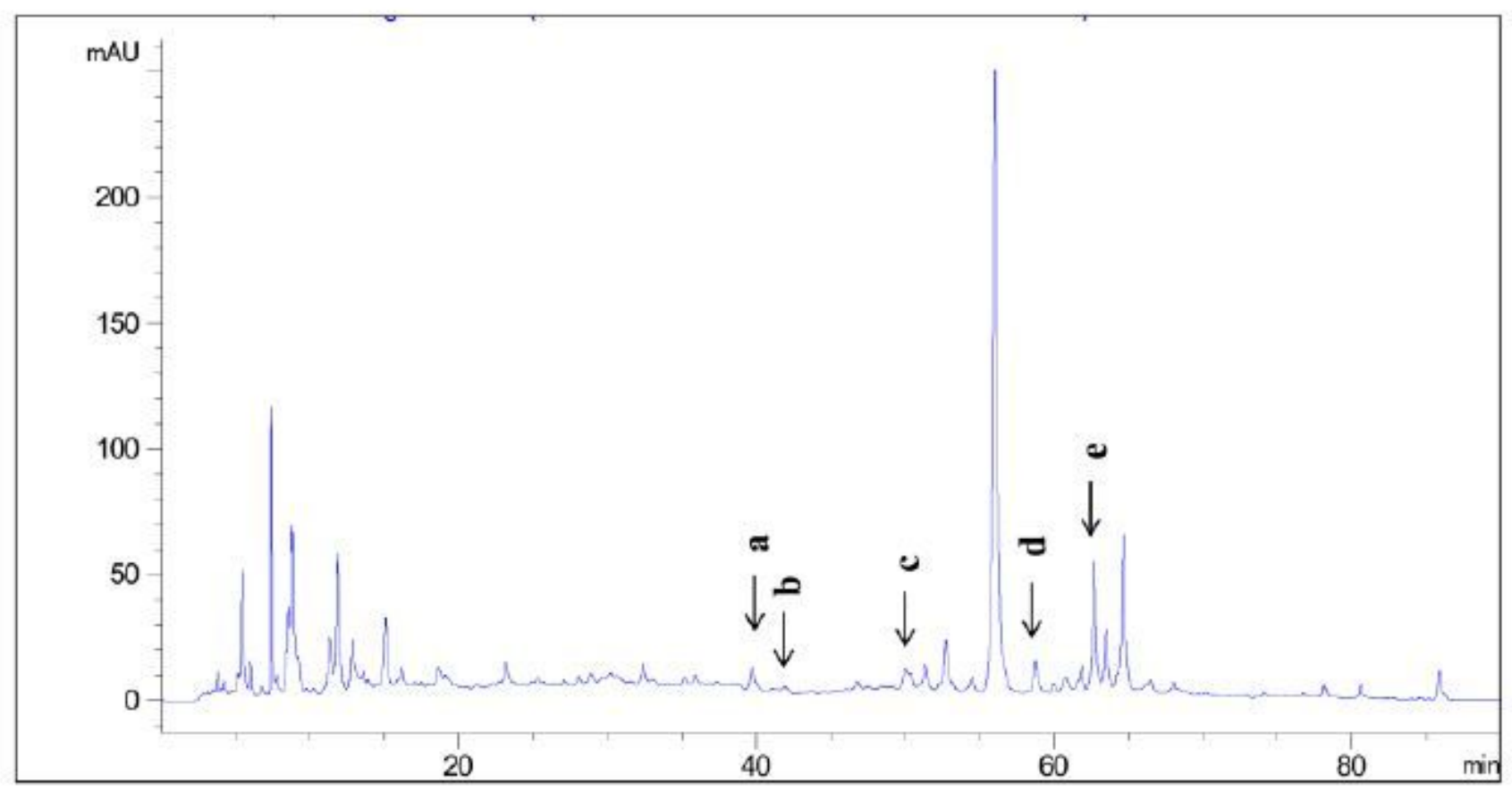

Figure 1

Study scheme and the fingerprinting of TJCYR. (A) Schematic diagram of experimental protocol; (B) Characteristic fingerprint of TJCYR was analyzed by HPLC. Pd, day of pregnancy; EID, embryo implantation dysfunction; TJCYR, Tiao Jing Cu Yun Recipe; HPLC, High Performance Liquid Chromatography; a, Calycosin-7-glucoside (standard substance for Astragalus membranaceus (Fisch.) Bunge); b, Acteoside (standard substance for Rehmannia glutinosa (Gaert.) Libosch.ex Fisch.et Mey.); c, Salvianolic acid B (standard substance for Salvia miltiorrhiza Bge.) ; d, Icariin (standard substance for Epimedium brevicornu Maxim.); e, Tanshinone IIA (standard substance for Salvia miltiorrhiza Bge.). 
A
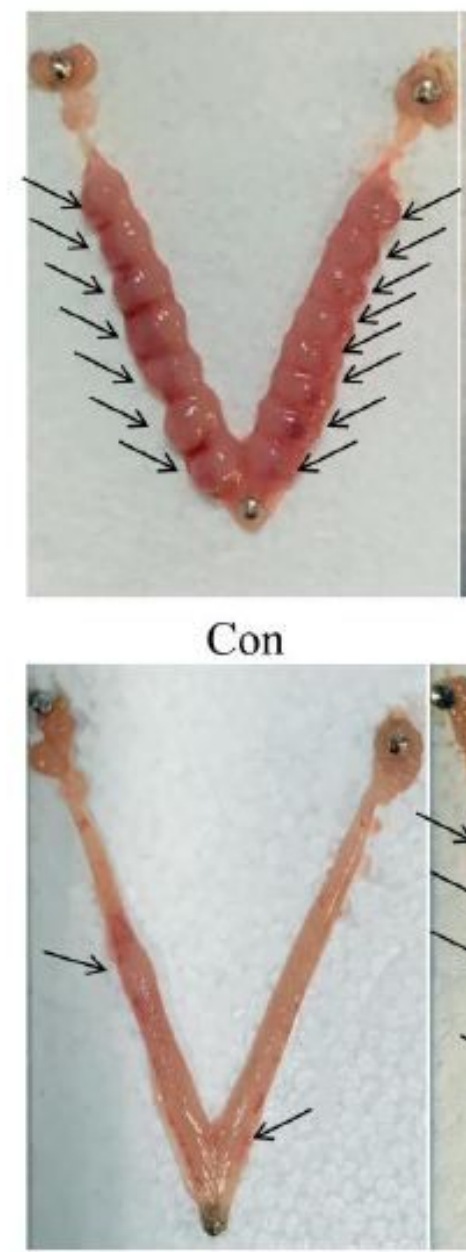

TJCYR-L+EDI

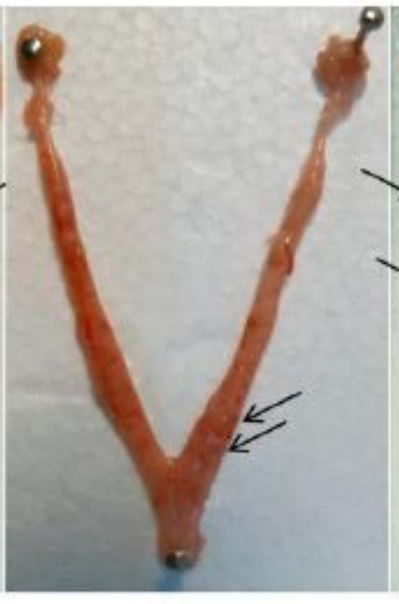

EDI
B

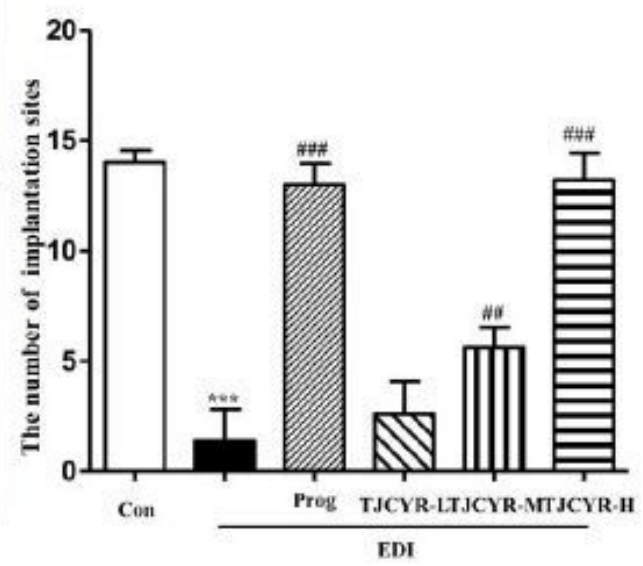

\section{Figure 2}

TJCYR incresed the number of implantation sites. (A) A representative photograph showing the number of implantation sites (the arrow pointed) at Pd8; (B) Quantification of implantation sites ( $n=8)$. Results are expressed as mean \pm SEM. ${ }^{*} * \mathrm{P}<0.001$ versus Control; \#\#P<0.05, \#\#\#P<0.01 versus EID only. EID, embryo implantation dysfunction; Prog, Progesterone; TJCYR, Tiao Jing Cu Yun Recipe; TJCYR-H, the high-dose TJCYR; TJCYR-M, the medium-dose TJCYR; TJCYR-L, the low-dose TJCYR. 
A

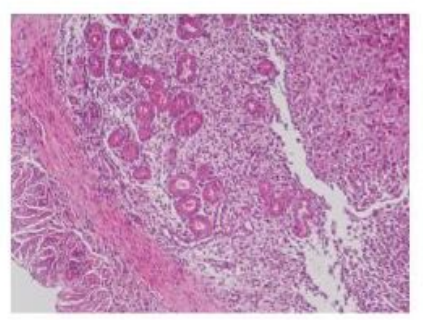

Control

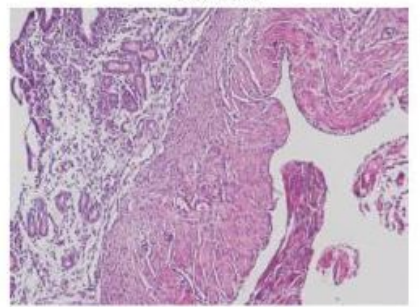

Prog+EID

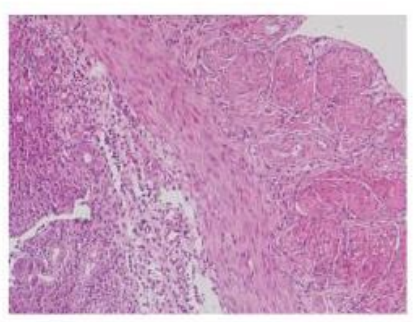

EID

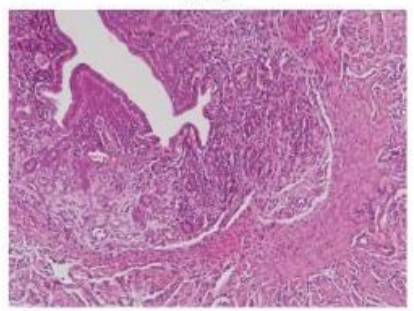

TJCYR+EID
B

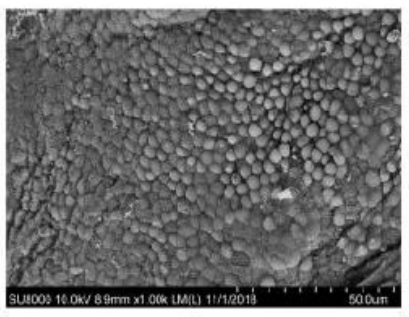

Control

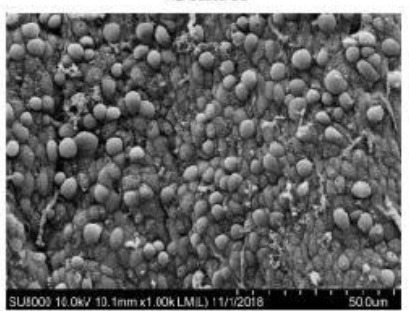

Prog+EID

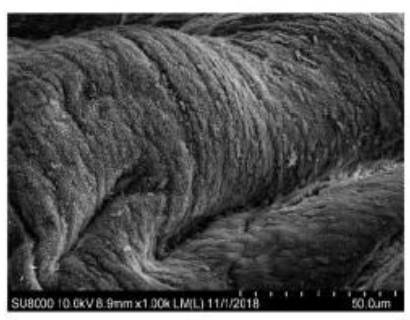

EID

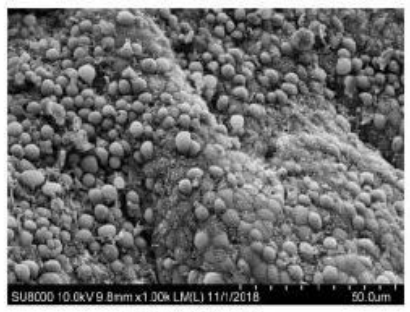

TJCYR+EID

\section{Figure 3}

Effect of TJCYR on EID-induced changes in endometrial morphology. (A) H\&E showing pathological changes in endometrium (×200); (B) SEM showing ultrastructure changes in pinopodes.

A

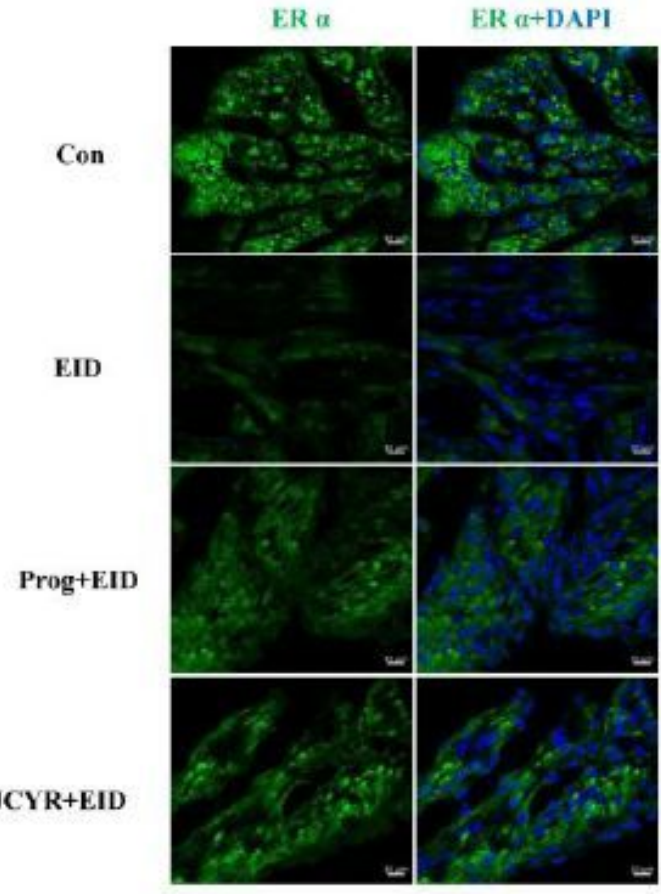

B

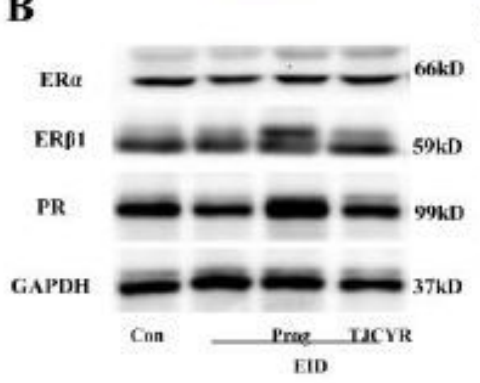

C

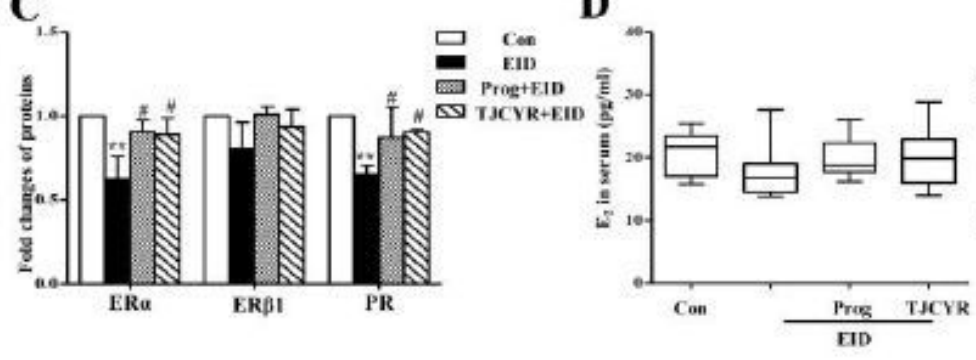

PR
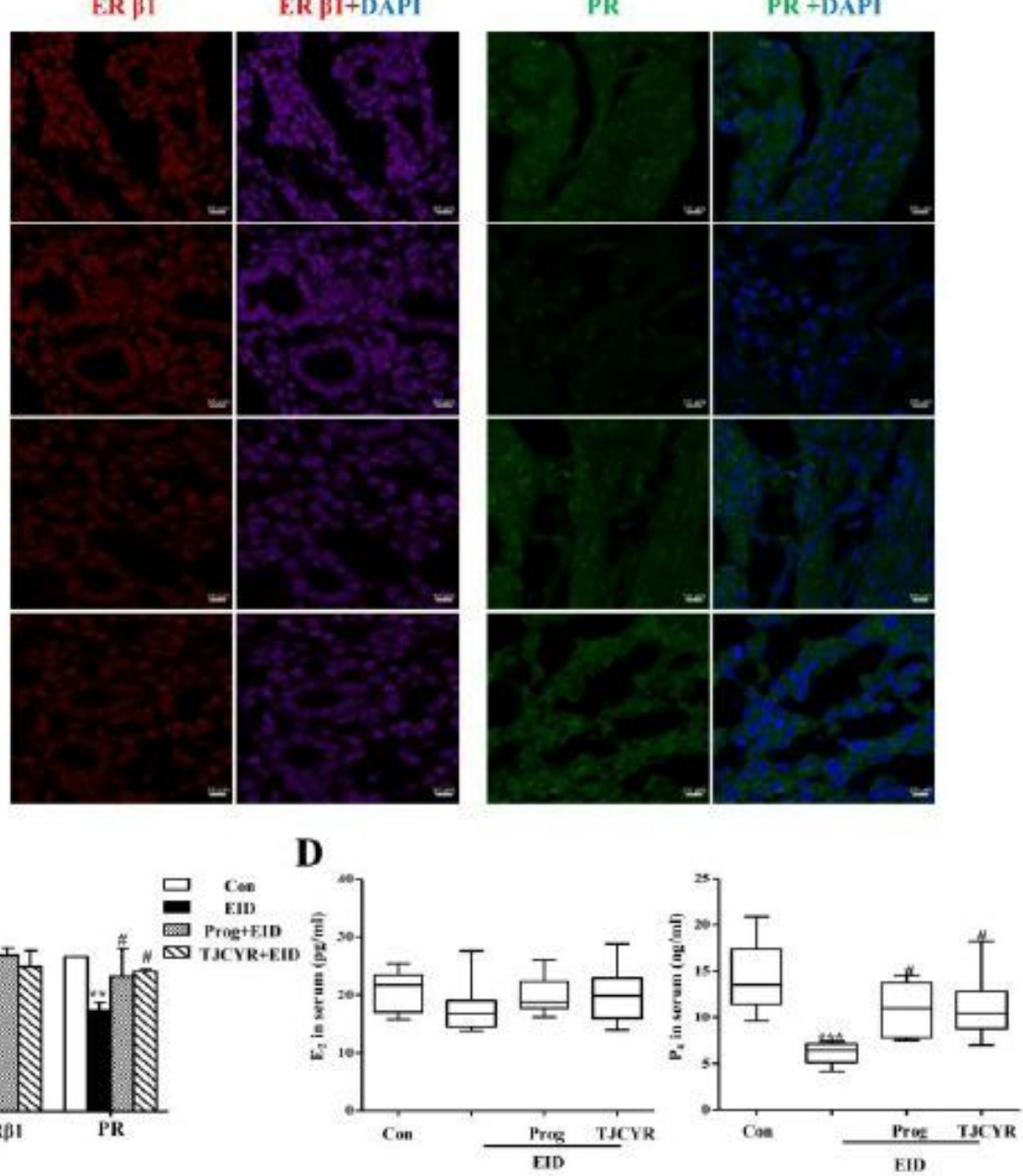


\section{Figure 4}

Effect of TJCYR on EID-induced changes in the hormones and receptors. (A) Representative confocal fluorescent images of the uterine sample with ERa (Green), ER $\beta 1$ (Red) and PR (Green), respectively. (B) Protein levels of ERa, ERß1 and PR in uterine tissue were determined by Western blotting ( $n=3)$; (C) Quantification of protein levels. (D) The level of E2 and P4 in serum were tested by radioimmunoassay. Results are expressed as mean \pm SEM. ${ }^{\star *}{ }^{*} P<0.001,{ }^{*} \mathrm{P}<0.01$ versus Control; $\# \mathrm{P}<0.05$ versus EID only. EID, embryo implantation dysfunction; Prog, Progesterone; TJCYR, Tiao Jing Cu Yun Recipe; E2, 17ß-estradiol; P4, progesterone; ERa, Estrogen Receptor alpha; ERß1, Estrogen Receptor beta1; PR, Progesterone Receptor.

A

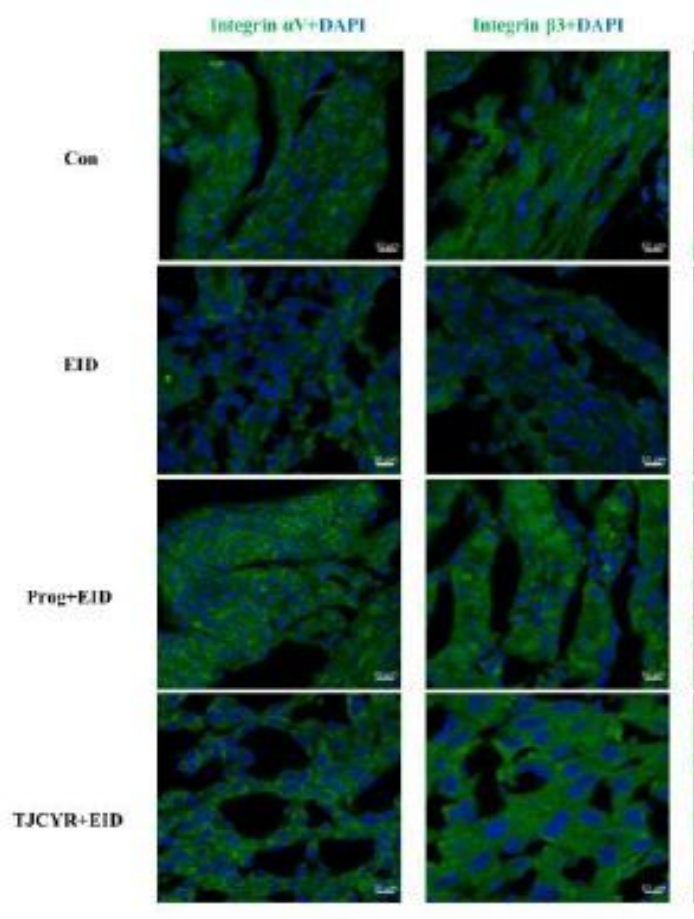

C

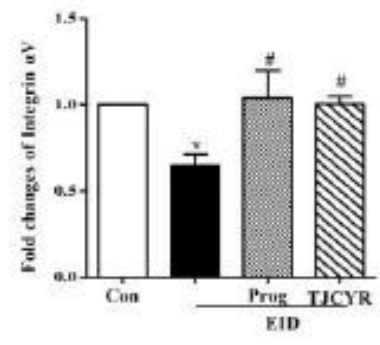

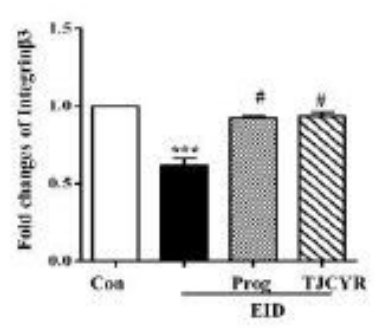

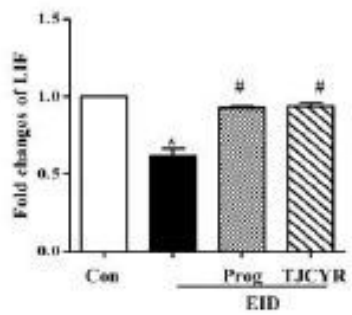

B
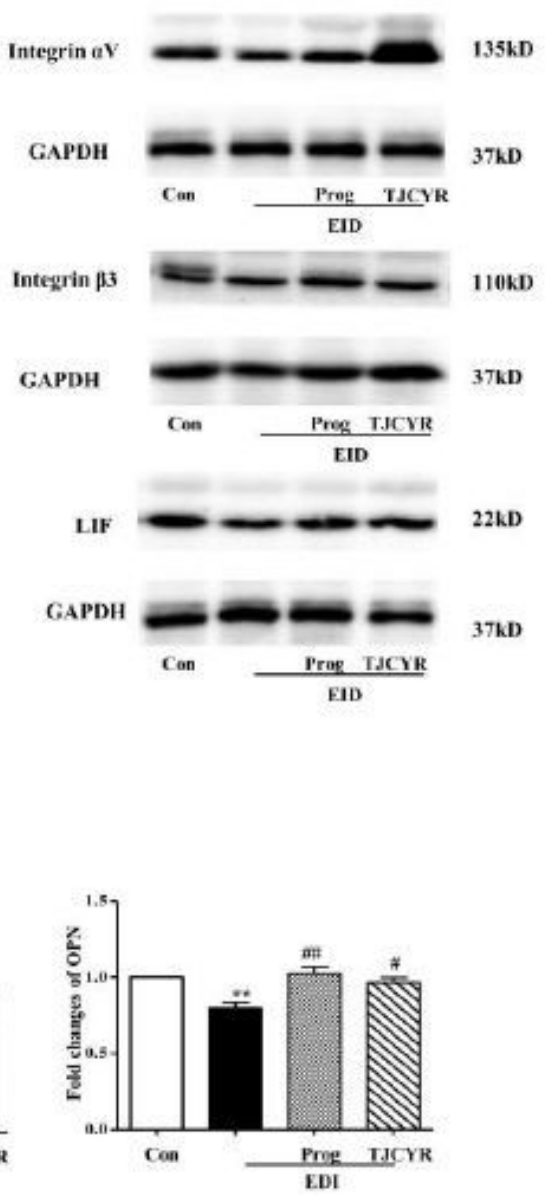

\section{Figure 5}

Effect of TJCYR on the biomarkers of endometrial receptivity. (A, D, G and J) Representative confocal fluorescent images of the uterine sample with Integrin $\mathrm{aV}$, Integrin $\beta 3$, LIF and OPN, respectively. $(B, E, G$ and K) Protein levels of Integrin $a V$, Integrin $\beta 3$, LIF and OPN in uterine tissue were determined by Western blotting ( $n=3) ;(C, F, I$ and $L)$ Quantification of protein levels. Results are expressed as mean \pm SEM. 


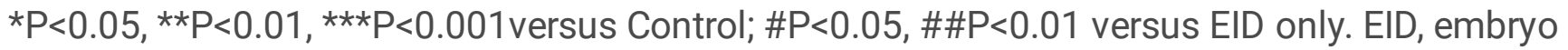
implantation dysfunction; Prog, Progesterone; TJCYR, Tiao Jing Cu Yun Recipe.

A

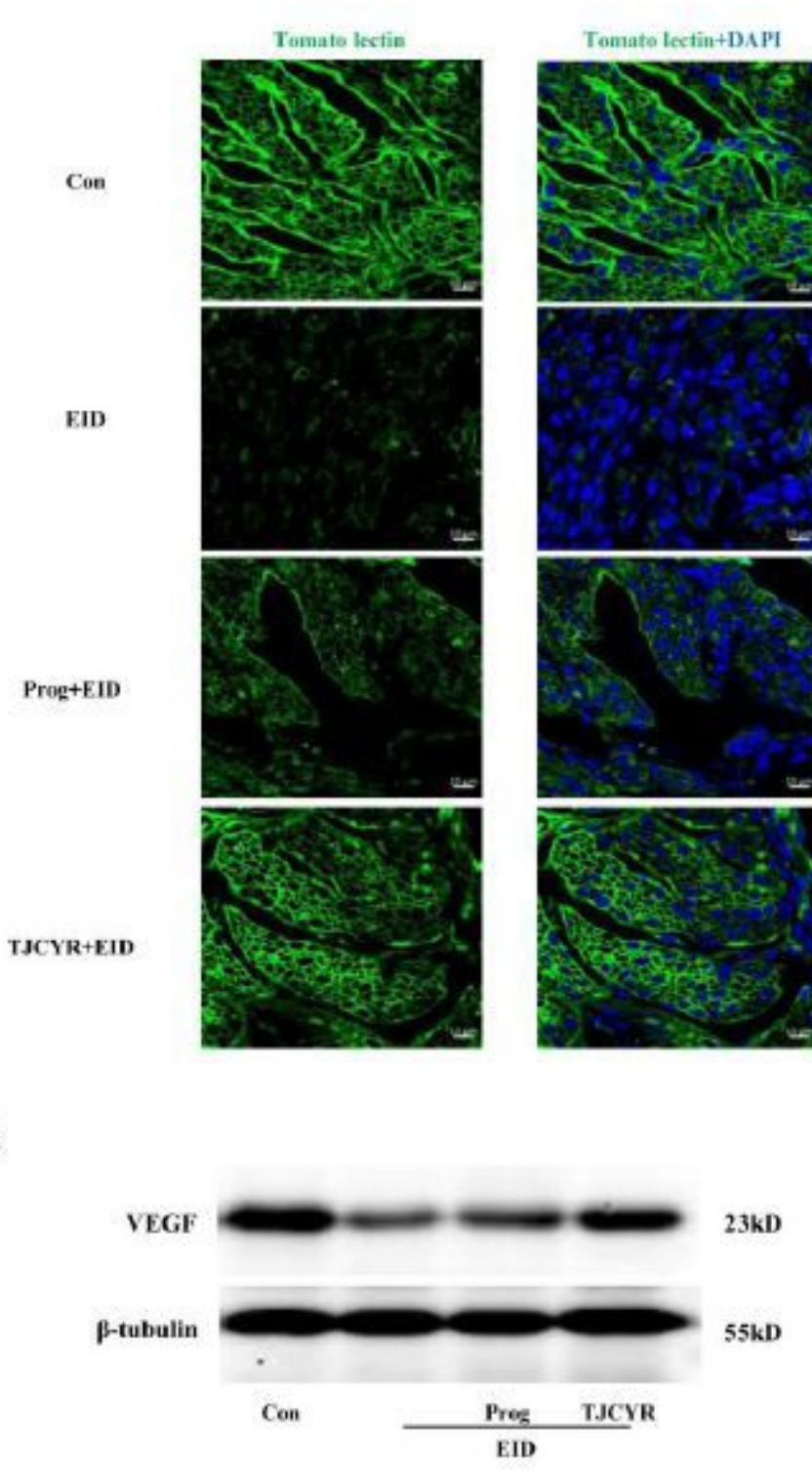

D

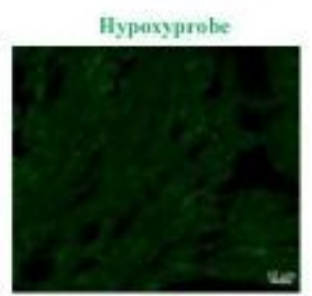

EID
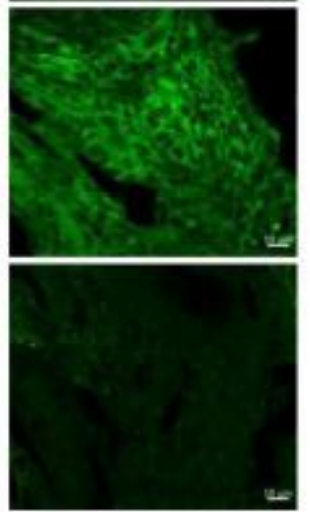

TJCYR+EID
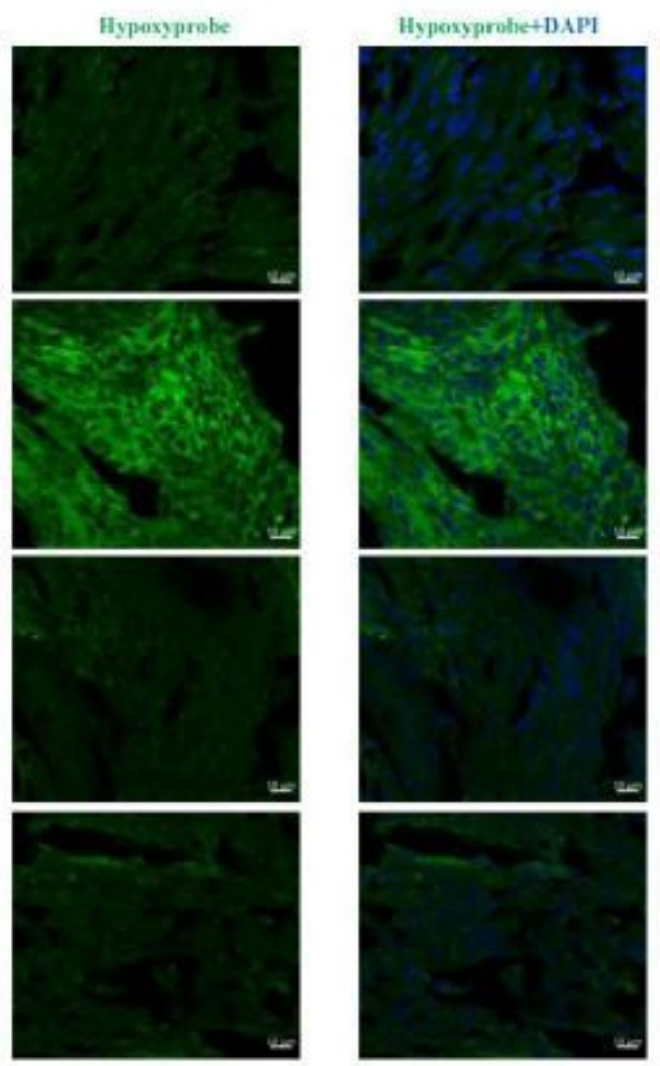

C

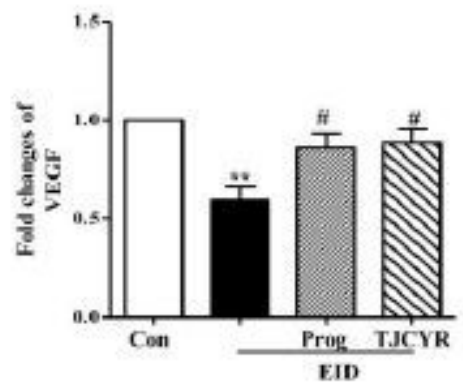

\section{Figure 6}

Effect of TJCYR on angiogenesis and hypoxia. (A) Representative confocal fluorescent images of the uterine sample with Tomato Lectin (Green) and DAPI (Blue); (B) Protein levels of VEGF in uterine tissue were determined by Western blotting $(n=3)$; (C) Quantification of protein levels. Results are expressed as mean \pm SEM; (D) Representative confocal fluorescent images of the uterine sample with Hypoxyprobe (Green) and DAPI (Blue). ${ }^{\star} \mathrm{P}<0.05,{ }^{*} \mathrm{P}<0.01$ versus Control; $\# \mathrm{P}<0.05$ versus EID only. EID, embryo implantation dysfunction; Prog, Progesterone; TJCYR, Tiao Jing Cu Yun Recipe. 
$\mathbf{A}$
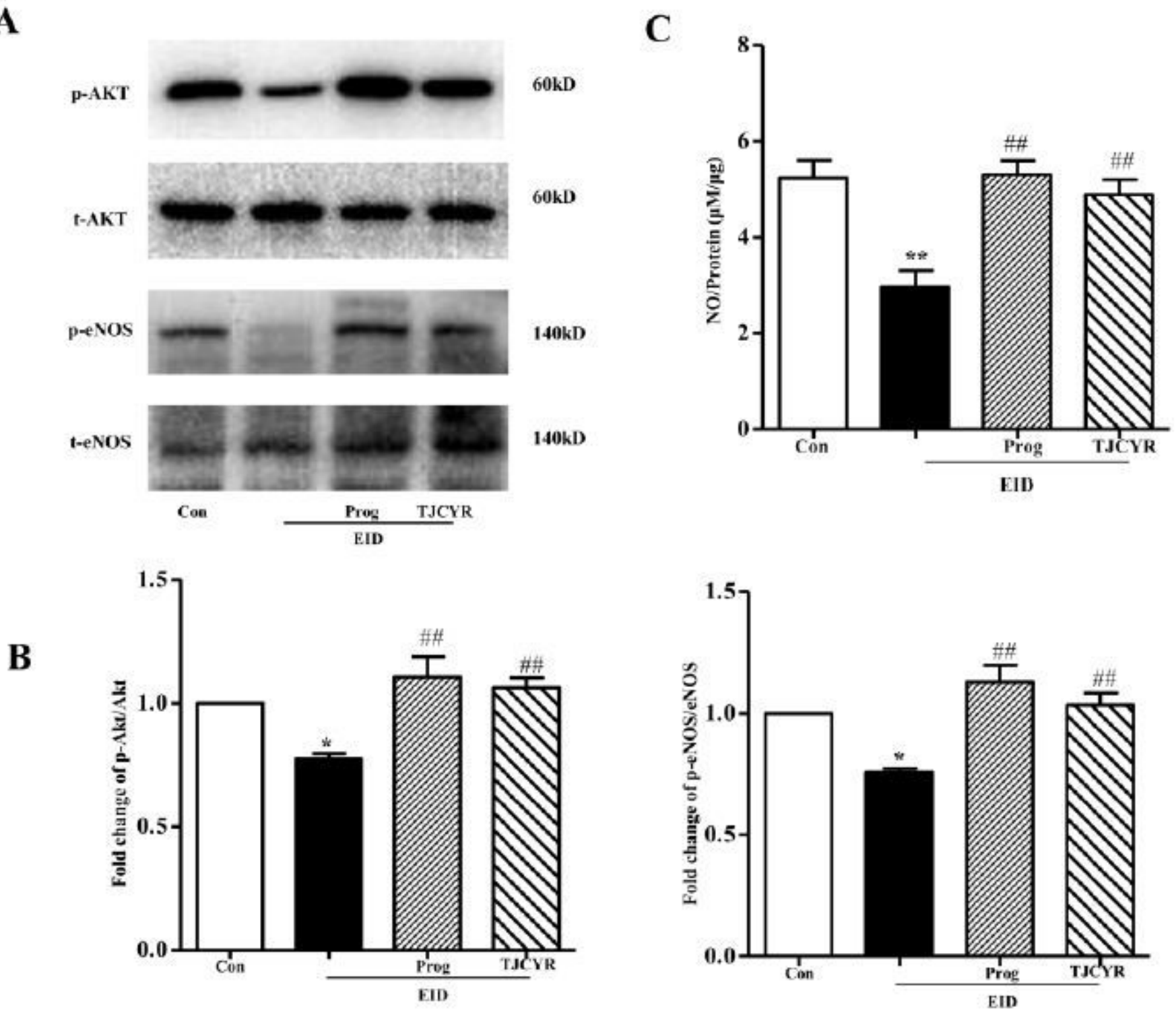

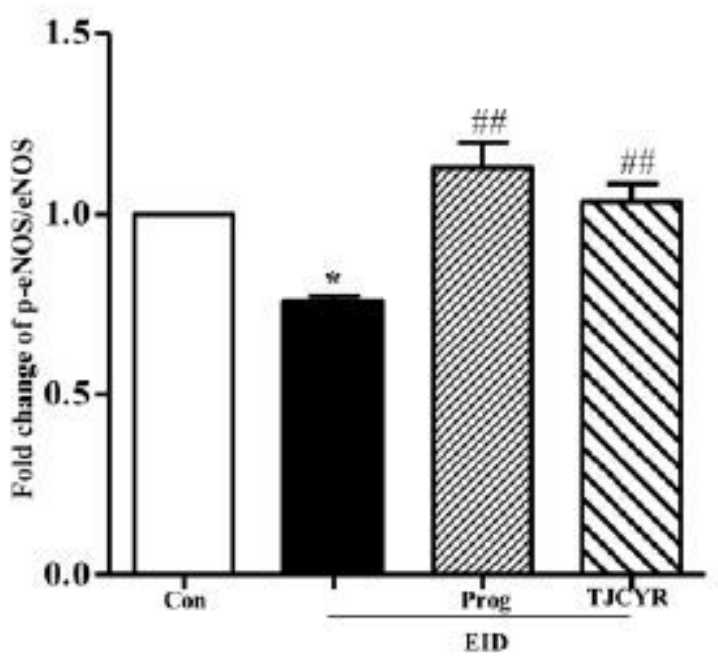

\section{Figure 7}

Effect of TJCYR on PI3K/Akt/eNOS signaling pathway. (A) Protein levels of PI3K/Akt/eNOS signaling pathway in uterine tissue were determined by Western blotting $(n=3)$; (B) Quantification of protein levels. Results are expressed as mean \pm SEM; (C) NO production was showed by NO/protein $(n=10)$. Results are expressed as mean \pm SEM. ${ }^{*} P<0.05$, ${ }^{\star *} P<0.01$ versus Control; \#\#P<0.01 versus EID only. EID, embryo implantation dysfunction; Prog, Progesterone; TJCYR, Tiao Jing Cu Yun Recipe; NO, nitric oxide. 